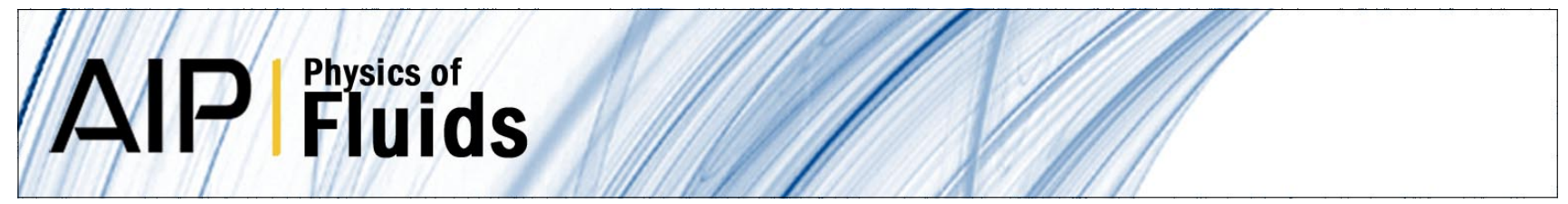

\title{
Large eddy simulation of smooth-wall, transitional and fully rough-wall channel flow
}

Namiko Saito, Dale I. Pullin, and Michio Inoue

Citation: Phys. Fluids 24, 075103 (2012); doi: 10.1063/1.4731301

View online: http://dx.doi.org/10.1063/1.4731301

View Table of Contents: http://pof.aip.org/resource/1/PHFLE6/v24/i7

Published by the American Institute of Physics.

\section{Related Articles}

Convection and reaction in a diffusive boundary layer in a porous medium: Nonlinear dynamics Chaos 22, 037113 (2012)

Symmetry analysis and self-similar forms of fluid flow and heat-mass transfer in turbulent boundary layer flow of a nanofluid

Phys. Fluids 24, 092003 (2012)

Detuned resonances of Tollmien-Schlichting waves in an airfoil boundary layer: Experiment, theory, and direct numerical simulation

Phys. Fluids 24, 094103 (2012)

Asymptotic expansion of the solution of the steady Stokes equation with variable viscosity in a two-dimensional tube structure

J. Math. Phys. 53, 103702 (2012)

Large-eddy simulation of turbulent channel flow using explicit filtering and dynamic mixed models

Phys. Fluids 24, 085105 (2012)

\section{Additional information on Phys. Fluids}

Journal Homepage: http://pof.aip.org/

Journal Information: http://pof.aip.org/about/about_the_journal

Top downloads: http://pof.aip.org/features/most_downloaded

Information for Authors: http://pof.aip.org/authors

\section{ADVERTISEMENT}

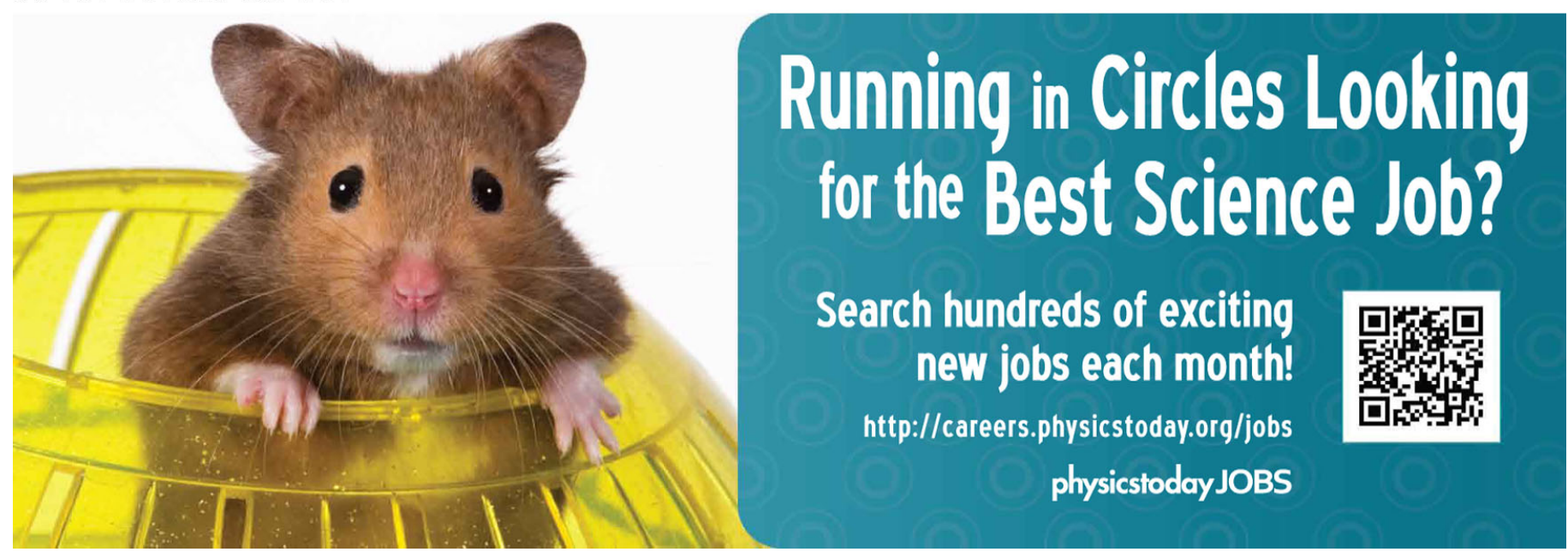




\title{
Large eddy simulation of smooth-wall, transitional and fully rough-wall channel flow
}

\author{
Namiko Saito, ${ }^{\text {a) }}$ Dale I. Pullin, and Michio Inoue \\ California Institute of Technology, Pasadena, California 91125, USA
}

(Received 20 February 2012; accepted 5 June 2012; published online 2 July 2012)

Large eddy simulation (LES) is reported for both smooth and rough-wall channel flows at resolutions for which the roughness is subgrid. The stretched vortex, subgridscale model is combined with an existing wall-model that calculates the local friction velocity dynamically while providing a Dirichlet-like slip velocity at a slightly raised wall. This wall model is presently extended to include the effects of subgrid wall roughness by the incorporation of the Hama's roughness function $\Delta U^{+}\left(k_{s \infty}^{+}\right)$that depends on some geometric roughness height $k_{s \infty}$ scaled in inner variables. Presently Colebrook's empirical roughness function is used but the model can utilize any given function of an arbitrary number of inner-scaled, roughness length parameters. This approach requires no change to the interior LES and can handle both smooth and rough walls. The LES is applied to fully turbulent, smooth, and rough-wall channel flow in both the transitional and fully rough regimes. Both roughness and Reynolds number effects are captured for Reynolds numbers $R e_{b}$ based on the bulk flow speed in the range $10^{4}-10^{10}$ with the equivalent $R e_{\tau}$, based on the wall-drag velocity $u_{\tau}$ varying from 650 to $10^{8}$. Results include a Moody-like diagram for the friction factor $f=f\left(\operatorname{Re}_{b}, \epsilon\right), \epsilon=k_{s \infty} / \delta$, mean velocity profiles, and turbulence statistics. In the fully rough regime, at sufficiently large $R e_{b}$, the mean velocity profiles show collapse in outer variables onto a roughness modified, universal, velocity-deficit profile. Outer-flow stream-wise turbulence intensities scale well with $u_{\tau}$ for both smooth and rough-wall flow, showing a log-like profile. The infinite Reynolds number limits of both smooth and rough-wall flows are explored. An assumption that, for smooth-wall flow, the turbulence intensities scaled on $u_{\tau}$ are bounded above by the sum of a logarithmic profile plus a finite function across the whole channel suggests that the infinite $R e_{b}$ limit is inviscid slip flow without turbulence. The asymptote, however, is extremely slow. Turbulent rough-wall flow that conforms to the Hama model shows a finite limit containing turbulence intensities that scale on the friction factor for any small but finite roughness. (c) 2012 American Institute of Physics. [http://dx.doi.org/10.1063/1.4731301]

\section{INTRODUCTION}

In the area of computational fluid dynamics, large eddy simulation (LES) has shown success in addressing the challenges of direct numerical simulation (DNS) associated with its high computational requirements. As the Reynolds number $(R e)$ is increased, the range of length scales existing in most turbulent flows increases rapidly, requiring correspondingly higher resolutions for DNS. The required grid points for DNS scales roughly as $O\left(R e^{9 / 4}\right)$, presently falling short of satisfying DNS needs at engineering Reynolds numbers. LES takes a different approach by resolving the larger scales while modeling the smaller scales. While LES of unbounded homogeneous and shear turbulence is well established (e.g., Misra and Pullin, ${ }^{1}$ Ferrante et al.,${ }^{2}$ Pitsch, ${ }^{3}$ Matheou et al.,${ }^{4}$ and Foysi and $\operatorname{Sarkar}^{5}$ ), LES of wall bounded flows has remained challenging, first, because the existence

\footnotetext{
a)namiko@caltech.edu.
} 
of a wall causes the length scales to be progressively smaller towards the wall, and second, owing to near-wall anisotropy. For near-wall resolved LES, these phenomena tend to bring the required computing resources up to $O\left(R e^{1.8}\right)$ (Piomelli'), close to that of DNS. However, the development of near-wall modeled LES (Refs. 6-11) has broadened the range of applications of LES for wall bounded flows. In near-wall modeled LES, the statistical effects of the near-wall anisotropic fine scales are not resolved but modeled via wall models that attempt to approximate near-wall physics. A summary of some current wall models is given by Inoue and Pullin. ${ }^{8}$ The progress of near-wall modeled LES techniques has made it possible to simulate wall bounded flows at Reynolds numbers of engineering interest. However, at sufficiently high Reynolds number, wall roughness effects emerge in experiments. Of notable example are the experimental studies in the Princeton Superpipe that are pushing their maximum Reynolds number above $38 \times 10^{6}$ (Marusic et al. ${ }^{12}$ ). In this facility, McKeon et al. ${ }^{13}$ recently confirmed that above $R e=20 \times 10^{6}$, even using advanced honing of pipes to create smooth-wall conditions, roughness effects can be detected. This suggests that roughness cannot be ignored in simulations like LES that aim to provide useful solutions in practice under high Reynolds number conditions.

Roughness effects were famously studied in pipes by Nikuradse,${ }^{14}$ introducing the hydrodynamic concept of sand grain roughness $k_{s}$ to represent the unique size of sand grains that were densely packed on the walls of pipes in his experiments. Colebrook ${ }^{15}$ found an empirical formula for the Darcy-Weisbach friction factor by incorporating industrial pipe data sets and Nikuradse's concept of sand roughness. Later Moody ${ }^{16}$ used the latter with Colebrook's formula to create the Moody diagram that illustrates the dependence of friction factor on both Reynolds number and relative roughness. This is a compelling visual of the four stages required to reach the fully rough limit as Reynolds number increases: laminar flow, transition to turbulent flow over a smooth wall, transitionally rough flow, and finally the fully rough asymptotic limit. Here, "fully rough" means that the Reynolds number is sufficiently high but the roughness height is small, $k / d \ll 1$, where $k$ is some length-scale measure of surface roughness and $d$ is a characteristic length scale.

A common choice for the roughness length scale $k$ is to denote it as the root-mean-square (r.m.s.) of the physical surface height profile $k(x, z)$ by $k_{R M S}$. Nikuradse developed another roughness length scale used commonly in later studies; he defined a parameter $k_{s}$ to represent the sand grain size he used to roughen pipe surfaces. Popular empirical models (e.g., Colebrook ${ }^{15}$ and Cebeci and Bradshaw ${ }^{17}$ ) that stem from Nikuradse's data sets make use of $k_{s}$ as an input, making it necessary for a non-sand grain rough surfaces to be characterized by an equivalent sand roughness. For any experiments or simulations using rough surfaces other than sand roughened walls, their roughness length scale is typically expressed as a "geometric" sand roughness $k_{s \infty}$, which is the size of sand grains from Nikuradse's experiments that are required to produce the same wall shear stress in the fully rough regime (Jiménez ${ }^{18}$ ). In what follows, we will characterize roughness by assigning nominal $k_{s \infty}$ values without considering the exact surface profile $k(x, z)$.

Later studies examined more closely the structure of wall bounded turbulent flow, especially in the canonical flows (pipes, channels, and zero-pressure-gradient boundary layers). When roughness is introduced, these flows admit to a classification of their behavior into three regimes depending on the roughness height. The first regime comprises smooth-wall flow. Roughness heights in the first regime range from $k^{+}=0$ to when $k^{+}$grows large enough to cause the onset of transitional roughness effects. Bradshaw ${ }^{19}$ highlighted the ongoing debate over which exact $k^{+}$represents the correct threshold for the onset of transition, and whether or not such a threshold even exists. Presently the upper limit of the first regime is considered in a broader sense as the roughness height $k^{+}$of the nominal wall surface for which the friction factor first departs from Prandtl's smooth-wall friction factor relation. ${ }^{20}$ In this smooth regime, the classical view of the smooth-wall structure is that the flow consists of a viscosity-dependent inner region and viscosity-independent outer region with the inner (viscous) length scale $v / u_{\tau}$ and outer length scale $\delta .{ }^{12}$ Here we denote molecular viscosity $v$, friction velocity $u_{\tau}=\sqrt{\left(\tau_{w} / \rho\right)}$, where $\tau_{w}$ and $\rho$ are the tangential wall shear stress and fluid density, respectively, and finally geometry length scale $\delta$ which represents pipe diameter, half-channel height, or boundary layer thickness.

The second regime comprises transitionally and fully rough flow. In the former, the friction factor exhibits dependence on Reynolds number whereas in the latter it is independent of Reynolds 
number. In terms of scaling, transitional flows reach the fully rough asymptote when $k^{+}$becomes large, thus indicating that the viscous length scale $v / u_{\tau}$ is no longer relevant compared to the roughness length scale. ${ }^{21}$ Commonly found in flows in the second regime is that the inner flow experiences a complex effect from wall roughness, and the outer flow only experiences a change through the roughness-modified wall shear stress. This is known as Townsend's hypothesis of outerlayer similarity (Townsend ${ }^{22}$ ). For smooth-wall flows, the velocity defect profile $u_{0}-u$, where $u_{0}$ is some outer variable, is known to collapse on $u_{\tau}$ and outer length scale $\delta$, as derived from the law of the wall by Millikan. ${ }^{12}$ Townsend's hypothesis then suggests that the same scalings should collapse this defect profile when roughness is introduced in the second regime. Schultz and Flack ${ }^{23}$ support Townsend's hypothesis through their experiments of the rough-walled boundary layer up to $R e_{\tau}$ $=10100$. Hama observed a momentum deficit in the outer layer mean-velocity profile caused by roughness-element pressure losses and that this was accompanied by a shift downward in the mean velocity profiles. A few exceptions to this observation include the experiments of Bechert et al. ${ }^{24}$ where the drag is observed to decrease for flow over ribbed surfaces causing an upward shift. To account for the aforementioned shift, Hama modified the log law to include an additive roughness function $\Delta U^{+}\left(\mathrm{Hama}^{25}\right.$ and Schultz and Flack $\left.{ }^{26}\right)$. Subsequent data has confirmed this shift (e.g., Shockling $e t ~ a l .{ }^{27}$ ) but not the functional dependence on $k_{s \infty}^{+}$, despite $\Delta U^{+}$being widely adopted. The debate surrounding this traces back to the inflectional profile of Nikuradse's friction factor compared to the monotonic profile of Colebrook during transition and it is on-going, as reviewed by Marusic et al. ${ }^{12}$

In the third regime, as the height $k$ of roughness elements grows to be extremely large, to a size suggested by Jiménez ${ }^{18}$ as being greater than $k / \delta \approx 1 / 40$, they penetrate well into the log law region thus affecting a significant portion of it and demarcating the start of the third regime. The result is a breakdown of similarity in the outer-layers. ${ }^{21}$ Presently we are not concerned with roughness of this final regime, but rather, from this point onwards consider only the smooth flow in the first regime and transitionally and fully rough flows in the second regime.

Although not the focus of this paper, we note that there exists a body of research concerned with finding universal length scales and understanding roughness topography effects, especially as it concerns $k_{s \infty}$. It is generally possible a posteriori to deduce $k_{s \infty}$ from a measured or simulated velocity profile. However, as stressed in Schultz and Flack, ${ }^{23}$ to use only geometric properties of the surface height distribution to predict $k_{s \infty}$ a priori remains an open problem of significant challenge and importance. Most prior efforts have been devoted to surfaces with roughness characterized by a single or few length scales. Schultz and Flack ${ }^{26}$ observed the collapse of nine single length scale pyramid covered surfaces exhibiting smooth-wall results upon plotting in velocity-defect form and supporting the use of similarity methods like Townsend's. Jiméne $z^{18}$ reviews the debate over roughness types, covering k-type and d-type surfaces (see also Leonardi et al. ${ }^{28}$ ), 2D surfaces and more. Also not the focus of this paper is the extensive work on geophysical flows viewed as rough surfaces, e.g., atmospheric boundary layers; see Monin, ${ }^{29}$ Counihan, ${ }^{30}$ and Metzger and Klewicki. ${ }^{31}$ Finally we note that the present paper will consider only the canonical flows without transition to turbulence or thermal effects.

There have been several DNS studies of rough-wall-bounded turbulent channel flows. ${ }^{32-35}$ Orlandi et al. ${ }^{32}$ considered 2D- and 3D-roughness elements which are $20 \%$ of their half channel height and are fully characterized by grid points on the elements. Their flow visualization provided insight into the complex flow structure near the roughness elements at $R e_{\tau}$ of a few hundred. Flores and Jiménez ${ }^{35}$ took a different approach by simulating rough channel flows at $R e_{\tau}=630$ where they replaced non-slip wall boundary conditions of smooth wall with zero-mean velocity disturbances and interpreted such perturbed velocity boundary conditions as the roughness effects. Their one-point statistics and spectral analysis of the flow properties including the mean velocity profiles confirmed Townsend's outer layer similarity hypothesis with an exception of the very large scales, which are known to correlate from the wall to the channel center. Additionally, they examined flows over an individual disturbance, revealing the extent of the roughness sublayer to be about six times the roughness height.

Only a few LES studies address surface roughness effects, e.g., Nakayama et al. ${ }^{36}$ and Anderson and Meneveau. ${ }^{37}$ The latter perform LES of open channel flow as a model of the atmospheric surface 
layer with multi-scaled surface roughness in the fully rough regime (mimicking naturally rough terrain). They search for a length scale parameter $\alpha$ such that the subgrid-scale (SGS) roughness height, $z_{0, \Delta}$ is proportional to the local root-mean-square of the unresolved part of height fluctuations, $\sigma$, by $z_{0, \Delta}=\alpha \sigma$. An appropriate value of $\alpha$ is found by requiring resolution-independence for the total drag force. Once the value of $\alpha$ is determined, it is used to calculate the unresolved SGS part of the stress boundary conditions for the LES, which is then combined with the resolved part of the stress boundary conditions. A scale-dependent Lagrangian dynamic model is implemented, where the Smagorinsky constant is calculated dynamically. In contrast to the traditional dynamic models, this model exhibits improved performance when applied to high-Reynolds number wall-bounded flows without refining the mesh near the walls. We note that since Anderson and Meneveau tailor their simulations for geophysical flows over natural terrain, they maintain interest only in the fully rough regime and neglect the viscous stress in the Navier-Stokes equations. Our present interest extends to the capturing of the transitionally rough regime as well. This work also contrasts with Anderson and Meneveau in that the viscosity or equivalently Reynolds number is fully quantified. Nakayama et al. ${ }^{36}$ compared post-filtered DNS with LES when both are performed on the same rough-wall geometry. Their results suggested that unresolved LES subgrid roughness can be accounted for with additional terms in the filtered momentum equation. An associated consequence is added computational cost to evaluate the extra terms at every grid point. This differs from our approach wherein the direct roughness effect is applied only in the wall model, or equivalently only within a half to one percent of the half channel height (at the present resolution), while the rest of the flow responds to the rough surfaces through the coupling between the outer LES and the wall model.

Presently, the roughness function $\Delta U^{+}$is incorporated directly into the wall model ${ }^{7}$ without altering the SGS model, ${ }^{1}$ reflecting physically how the roughness is essentially viewed as an additional drag that modifies the wall boundary condition on the outer flow. In particular, channel flows with subgrid roughness are simulated in the fully developed turbulent regime. With a choice of the Colebrook formula for the roughness function, we capture the flow in both transitionally and fully rough regimes in terms of the friction factors, mean velocity profiles, turbulent statistics, and dissipations. In what follows, we will first present a summary of the derivation of the stretched vortex model and the wall model (Misra and Pullin, ${ }^{1}$ Chung and Pullin, ${ }^{7}$ and Inoue and Pullin ${ }^{8}$ ) in Sec. II, followed by the extension of this approach to surface roughness in Sec. II D. Sections IIIVI describe some results and discussion of the LES, followed by conclusions in Sec. VII.

\section{SGS MODEL AND WALL MODEL WITH ROUGHNESS}

The implementation of the LES for a rough channel comprises two major parts, the stretched vortex SGS model and the wall model. The former is unaffected by the introduction of roughness whereas the latter needs to be modified to incorporate the roughness model. In this section, an overview of the SGS model is given, followed by the extension of the wall model (Chung and Pullin $^{7}$ ) to include surface roughness. At this point, the wall model with roughness is developed with a generic form of roughness correction. We then proceed to discuss the selection of the specific form of roughness function used presently from numerous roughness functions available for other flows and applications.

\section{A. Stretched vortex SGS model}

The stretched vortex approach is a structural SGS model designed to represent the statistical effect of subgrid motion by using information from resolved scale quantities. ${ }^{1}$ It is assumed that the subgrid vorticity in each cell comprises a superposition of stretched vortices, each unidirectional and of "cylindrical" type. Upon coordinate transformation from the vortex-fixed frame to the lab-fixed frame, the distribution of orientations of the vortex structures forms a probability density function (PDF) which reflects the local anisotropy of the turbulence (Pullin and Saffman ${ }^{38}$ ). Extending the assumption that the ensemble dynamics of subgrid scale motion are dominated by a vortex aligned with a unit vector $e^{v}$, modeled via a delta-function PDF, the subgrid stress tensor is given by (Pullin 
and Saffman ${ }^{38}$ and Chung and Pullin ${ }^{7}$ )

$$
T_{i j}=\left(\delta_{i j}-e_{i}^{v} e_{j}^{v}\right) K .
$$

Here in (1), the subgrid stress tensor, $T_{i j}=\widetilde{u_{i} u_{j}}-\widetilde{u_{i}} \widetilde{u_{j}}$ is expressed in terms of the unit vector, $e^{v}$ and the subgrid kinetic energy $K$, which is given by the integral of the subgrid stress energy spectrum $E(k)$ as

$$
K=\int_{k_{c}}^{\infty} E(k) d k
$$

where $k_{c}=\pi / \Delta_{c}$ is the cut-off wave number and $\Delta_{c}=\left(\Delta_{x} \Delta_{y} \Delta_{z}\right)^{1 / 3}$. In what follows, the derivation of (1) is reviewed in more detail.

For subgrid stress closure, the subgrid kinetic energy $K$ must be obtained as in (2). The energy spectrum for turbulent incompressible flow $E(k)$, an essential part of calculating the subgrid kinetic energy, is known to have the asymptotic solution of the form (3) below for large wave number $k$, where $\epsilon$ is the dissipation rate per unit mass and $\eta$ is the Kolmogorov length. This relation was initially obtained by using dimensional reasoning by Kolmogorov, and later derived from the Navier Stokes equation by Lundgren ${ }^{39}$ in the form of (4) for stretched spiral type vortices, where $\bar{a}=e_{i}^{v} e_{j}^{v} S_{i j}$ is the stretching along the subgrid vortex axis exerted by the resolved scales, and $S_{i j}$ is the resolved strain-rate tensor

$$
\begin{aligned}
E(k) & =\epsilon^{2 / 3} k^{-5 / 3} F(\eta k), \\
& =\mathcal{K}_{0} \epsilon^{2 / 3} k^{-5 / 3} \exp \left(-\frac{2 k^{2} v}{3|\bar{a}|}\right) .
\end{aligned}
$$

Upon integration of (4) in accordance with (2), the subgrid kinetic energy is obtained as (5) in terms of a group constant $\mathcal{K}_{0}^{\prime}$ and an incomplete gamma function:

$$
K=\frac{1}{2} \mathcal{K}_{0}^{\prime} \Gamma\left[-\frac{1}{3}, \kappa_{c}^{2}\right], \text { where } \Gamma[s, t]=\int_{t}^{\infty} u^{s-1} \exp (-u) d u .
$$

Here, $\mathcal{K}_{0}^{\prime}=\mathcal{K}_{0} \epsilon^{2 / 3} \lambda_{v}^{2 / 3}, \lambda_{v}=(2 v / 3|\bar{a}|)^{1 / 2}$, and $\kappa_{c}=k_{c} \lambda_{v}$. The approximation of $\Gamma[s, t]$ and the evaluation of $\mathcal{K}_{0}^{\prime}$ are given in Chung and Pullin. ${ }^{7}$

Chung and Pullin ${ }^{7}$ extended (1) to incorporate the transport of resolved-scale axial velocity, modeled as a passive scalar, by SGS vortices as ${ }^{40,41,42,7}$

$$
\begin{aligned}
T_{i j} & =\widetilde{u_{i}^{\prime} u_{j}^{\prime}}+\widetilde{\widetilde{u_{i}^{\prime}} \widetilde{u_{j}^{\prime}}}+\widetilde{\widetilde{u_{i}^{\prime} u_{j}^{\prime}}}, \\
& =K\left(\delta_{i j}-e_{i}^{v} e_{j}^{v}\right)-K_{s}\left\{e_{j}^{v} e_{k}^{v} \frac{\partial \widetilde{u_{k}}}{\partial x_{l}}\left(\delta_{l i}-e_{l}^{v} e_{i}^{v}\right)+e_{i}^{v} e_{k}^{v} \frac{\partial \widetilde{u_{k}}}{\partial x_{l}}\left(\delta_{l j}-e_{l}^{v} e_{j}^{v}\right)\right\},
\end{aligned}
$$

where $2 K_{s}=\gamma \Delta_{c} K^{1 / 2}$ and $\gamma$ is a momentum mixing constant. This extended version of the shear stress is utilized subsequently to obtain a slip velocity in the implementation of the wall model.

\section{B. Wall model with roughness: Friction velocity}

The challenge in the LES of wall bounded flows is that the turbulent length scales become progressively smaller towards the wall due to confinement of the near-wall eddies. In near-wall resolved LES this is addressed by introducing a non-uniform mesh that has higher mesh refinement near the wall, and thus capturing the near-wall fine scales. In wall-modeled LES the near-wall anisotropic fine scales are modeled via a wall model, thus eliminating the need for higher grid refinement. Chung and Pullin ${ }^{7}$ define a slip velocity at a lifted virtual wall, thus providing the outer LES with slip Dirichlet boundary conditions. This wall model is presently extended to rough surfaces while retaining two major features, first solving an ordinary differential equation (ODE) to obtain the friction velocity and subsequently evaluating the slip velocity utilizing Townsend's attached-eddy hypothesis. We have denoted $x, y$, and $z$ as stream-wise, wall-normal, and span-wise coordinates, 
respectively, and $u, v$, and $w$ are the corresponding velocity components. The wall-parallel filtering and wall-normal integration operators are defined by

$$
\begin{gathered}
\tilde{\phi}(x, y, z, t)=\iint \phi\left(x^{\prime}, y, z^{\prime}, t\right) G\left(x-x^{\prime} ; \Delta_{f}\right) G\left(z-z^{\prime} ; \Delta_{f}\right) d x^{\prime} d z^{\prime}, \\
\langle\widetilde{\phi}\rangle(x, z)=\frac{1}{h-k(x, z)} \int_{k(x, z)}^{h} \tilde{\phi}(x, y, z) d y,
\end{gathered}
$$

where $\widetilde{\phi}$ denotes wall-parallel filtering and $\langle\phi\rangle$ denotes a wall-normal average and $\Delta_{f}$ is the filter width much smaller than the viscous length scale.

We proceed to give a heuristic derivation of an ODE for the friction velocity $u_{\tau}$ for the roughwall flow. Let the wall shape be $y=k(x, z)$ with $\overline{k(x, y)}=0$ and define $f(x, y, z)=k(x, z)-y$. Here, we denote $\overline{(~)}$ as the average over the wall-parallel plane. The wall-normal (into the wall) is $\mathbf{n}=$ $\nabla f /|\nabla f|$ on $f=0$. We consider a local control volume at the channel wall with $x, z$ dimensions $\Delta x$, $\Delta z$ (the local grid size), and $y$-dimension an arbitrary thickness $h$. It is further assumed that $\Delta x, \Delta z$, and $h$ are all much larger than $\max |k(x, z)|$. The four wall-normal surfaces intersect the wall and the bottom surface is the wall itself. Applying top-hat filtering and averaging defined above to the momentum equations, we obtain an integral form of the stream-wise momentum equation over the control volume

$$
\frac{\partial}{\partial t} \iiint \mathbf{u} d V=-\oiint \mathbf{n} \cdot\left(\mathbf{u} \mathbf{u}+\frac{p}{\rho} \mathbf{I}-\frac{\boldsymbol{\tau}}{\rho}\right) d S,
$$

where $\boldsymbol{\tau}=2 \nu \mathbf{S}$ and $\mathbf{S}$ is the strain-rate tensor. The RHS of (9) essentially represents the flux of momentum though the planes defining the control volume, and exterior force on the control volume consists of the convective, pressure, and viscous terms with each contribution requiring special wall boundary treatment upon our inclusion of rough walls.

The pressure term evaluated at the rough wall is non-zero due to the variation of $\mathbf{n}$. Since the roughness geometry of interest is subgrid, this pressure contribution from the rough wall introduces an unknown term in the filtered-averaged momentum equation. A similar situation is found for the viscous contribution at the rough wall, which is also in the form of surface integral over an unknown surface geometry. Collecting all the flux contributions including unknown terms, and approximating flux differences across wall-normal surfaces in terms of wall-parallel derivatives, the cell-averaged stream-wise momentum equation for the control volume is rewritten as

$$
\begin{aligned}
& \frac{\partial\langle\widetilde{u}\rangle}{\partial t}+\frac{\partial\langle\widetilde{u u}\rangle}{\partial x}+\frac{\partial\langle\widetilde{u w}\rangle}{\partial z}+\left.\frac{1}{h} \widetilde{u v}\right|_{h} \\
& \quad=-\left.\frac{\partial \widetilde{P}}{\partial x}\right|_{h}+\left.\frac{v}{h} \frac{\partial \widetilde{u}}{\partial y}\right|_{h}-\frac{1}{h}\left[\frac{1}{\Delta x \Delta z} \iint_{w} P n_{x} d S-\frac{v}{\Delta x \Delta z} \iint_{w} \mathbf{n} \cdot \nabla \mathbf{u} d S\right],
\end{aligned}
$$

where $P=p / \rho, n_{x}$ is the stream-wise component and the surface integral $\iint_{w}$ denotes the integral over the wall. The last two integral terms are the unknown pressure and viscous terms due to roughness. To encapsulate the wall skin friction contributions, we define the friction velocity $u_{\tau}$ as

$$
u_{\tau}^{2}=\frac{1}{\Delta x \Delta z} \iint_{w} P n_{x} d S-\frac{v}{\Delta x \Delta z} \iint_{w} \mathbf{n} \cdot \nabla \mathbf{u} d S=\frac{1}{\rho} \overline{\tau_{w}},
$$

where $\overline{\tau_{w}}$ is the surface drag force per unit area and $\overline{()}$ refers to an average over the intersection of the control volume (cell) and the wall. The differential equation (10) then becomes

$$
\frac{\partial\langle\widetilde{u}\rangle}{\partial t}+\frac{\partial\langle\widetilde{u u}\rangle}{\partial x}+\frac{\partial\langle\widetilde{u w}\rangle}{\partial z}+\left.\frac{1}{h} \widetilde{u v}\right|_{h}=-\left.\frac{\partial \widetilde{P}}{\partial x}\right|_{h}+\left.\frac{v}{h} \frac{\partial \widetilde{u}}{\partial y}\right|_{h}-\frac{1}{h} u_{\tau}^{2}
$$

Equation (12) is identical to the smooth-wall case (see Chung and Pullin ${ }^{7}$ ) but contains a generalized definition of the friction velocity to account for the additional pressure drag and corrected viscous contributions to the total surface drag for rough walls. The smooth-wall case with the conventional definition of $u_{\tau}$ is recovered with $k(x, y)=0$ and $\mathbf{n}=(0,1,0)$. 


\section{Inner scaling}

The unsteady term of (12) is treated with a general form of inner scaling. A classical but empirical roughness correction to inner scaling accounts for an increased momentum deficit due to the surface drag on the roughness elements, resulting in a downward shift of the inner-scaled mean velocity profile (Schultz and Flack ${ }^{26}$ ). Such a downward shift is quantified via roughness function, $-\Delta U^{+}$which is incorporated in the inner scaling as

$$
\widetilde{u}=u_{\tau}\left(F_{1}\left(y^{+}\right)-\Delta U^{+}\left(k_{s \infty}^{+}\right)\right),
$$

where $y^{+}=y u_{\tau} / \nu\left(\right.$ Jiménez $\left.^{18}\right)$ and we take $F_{1}(0)=0$. The first term $F_{1}\left(y^{+}\right)$is the common term for both smooth and rough walls while $-\Delta U^{+}\left(k_{s \infty}^{+}\right)$is the roughness function expressed in terms of geometric roughness, $k_{s \infty}$ and $k_{s \infty}^{+}=k_{s \infty} u_{\tau} / \nu$. Applying the wall-parallel filter, wall-normal average, and temporal derivative, we obtain the expression given by

$$
\frac{\partial}{\partial t}\langle\tilde{u}\rangle=\frac{d}{d t}\left(\frac{u_{\tau}}{h} \int_{0}^{h} F_{1}\left(y^{+}\right)-\Delta U^{+}\left(k_{s \infty}^{+}\right) d y\right),
$$

where the friction velocity is spatially and temporally variant, i.e., $u_{\tau}=u_{\tau}(x, z, t)$. Performing the time derivative then gives

$$
\frac{\partial}{\partial t}\langle\tilde{u}\rangle=\frac{\partial u_{\tau}}{\partial t}\left(\frac{\left.\tilde{u}\right|_{h}}{u_{\tau}}-k_{s \infty}^{+} \frac{\partial \Delta U^{+}}{\partial k_{s \infty}^{+}}\right) .
$$

Note that integrals of $F_{1}\left(y^{+}\right)$do not appear in this expression owing to cancellation.

Substitution of (15) into the averaged-filtered momentum equation (12) and approximations of the filtered-averaged nonlinear terms as values at $y=h$ (one-point estimates), we obtain an ODE for $u_{\tau}$ as

$$
\frac{d u_{\tau}}{d t}=\frac{-\left.\frac{\partial \widetilde{u u}}{\partial x}\right|_{h}-\left.\frac{\partial \widetilde{u w}}{\partial z}\right|_{h}-\left.\frac{\partial \widetilde{P}}{\partial x}\right|_{h}-\left.\frac{1}{h} \widetilde{u v}\right|_{h}+\left.\frac{v}{h} \frac{\partial \widetilde{u}}{\partial y}\right|_{h}-\frac{1}{h} u_{\tau}^{2}}{\frac{\left.\widetilde{u}\right|_{h}}{u_{\tau}}-k_{s \infty}^{+} \frac{\partial \Delta U^{+}}{\partial k_{s \infty}^{+}}} .
$$

The height $h$ protrudes to the first or second cell of the LES domain and all filtered quantities on the right-hand side are provided by the LES itself. The presence of surface roughness is apparent in the denominator of (16) in the form of the derivative of $\Delta U^{+}$, which indicates that the roughness function is dependent on the inner-scaled roughness height and that the friction velocity is calculated dynamically at each wall point. Equation (16) allows local dynamical calculation of $u_{\tau}$. In practice, an equivalent differential equation for $\eta=u_{\tau}^{2} / \nu$ is solved. ${ }^{7,8}$ While for the present channel flow, the wall-averaged $u_{\tau}$ can be calculated using an integrated pressure-gradient, wall-drag balance (essentially an extension of the above argument to the whole channel), (16) is more general and can be used for boundary layer flows in the presence of pressure gradient. Further formal extensions of (16) to three-dimensional boundary layers in the presence of wall curvature are straightforward as are the incorporation of more general roughness functions which are functions of several roughness scales $\Delta U^{+}\left(k_{s \infty}^{+(1)}, k_{s \infty}^{+(2)}, k_{s \infty}^{+(3)}, \ldots\right)$.

\section{Wall model with roughness: Slip velocity at a lifted, flat virtual wall}

Chung and Pullin ${ }^{7}$ defined three near-wall regions: (I) $0<y<h_{\nu}$, essentially the viscous sublayer and part of the buffer layer, (II) $h_{v}<y<h_{0}$, part of the overlap layer where the shear stress is approximately constant and is modeled by the extended stretched vortex SGS model consisting of attached vortices aligned with the stream-wise direction, (III) $h_{0}<y$, where non-universal outer flow features are computed by LES coupled with the original stretched vortex SGS model of detached subgrid vortices aligned with the most extensive strain-rate direction. The lifted virtual wall refers to a plane at $y=h_{0}$, somewhere within the overlap region. In region (I), the velocity shows a linear profile, i.e., $\widetilde{u^{+}}=y^{+}$where $\widetilde{u^{+}}=\widetilde{u} / u_{\tau}$ and $y^{+}=y u_{\tau} / \nu$. We take $h_{v}^{+}$to be the intercept of the linear 
and empirical log-profile of the law of the wall, which is $h_{v}^{+} \approx 11$ for a hydrodynamically smooth wall.

A summary of the derivation of the slip velocity is presented by Inoue and Pullin. ${ }^{8}$ The derivation begins by assuming that the total shear stress is approximately constant (Townsend ${ }^{22}$ ) and that in the overlap region (region II) near-wall vortices are stream-wise aligned (e.g., Head and Bandyopadhyay ${ }^{43}$ and Robinson ${ }^{44}$ ). Such conditions allow us to reduce the Reynolds shear stress (6) to

$$
T_{x y}=-\frac{1}{2} \gamma_{\mathrm{II}} K^{1 / 2} \Delta_{c} \frac{d \tilde{u}}{d z} .
$$

Now, we further assume that the near-wall region can be modeled by a hierarchy of stream-wise aligned vortices whose scales are proportional to the wall distance (Chung and Pullin ${ }^{7}$ ). Along with (17), we arrive at

$$
\frac{d \tilde{u}}{d y}=\frac{1}{\mathcal{K}_{1}} \frac{u_{\tau}}{y}, \text { where } \mathcal{K}_{1}=-\frac{\gamma_{\text {II }} K^{1 / 2}}{2\left(-T_{x y} / u_{\tau}\right)} .
$$

The idea is called attached eddies (Nickels et $a l .{ }^{45}$ ) as opposed to detached eddies existing in the outer part of the flow where the vortices are unaware of the wall. Upon integration of (18) and setting the constant of integration by requiring that the log law intersects with the linear-relation near the wall at a particular distance from the wall, $y^{+}=h_{v}^{+}\left(\right.$Chung and Pullin $\left.{ }^{7}\right)$, a log-relation is obtained for the velocity in the overlap layer above smooth walls as

$$
\tilde{u}=u_{\tau}\left(\frac{1}{\mathcal{K}_{1}} \log \left(y^{+}\right)+B\right),
$$

where $B=h_{v}^{+}-\mathcal{K}_{1}^{-1} \log \left(h_{v}^{+}\right)$. The extension of this relation to rough surfaces is carried out through the application of the roughness correction $\Delta U^{+}\left(k_{s}^{+}\right)$to this relation. Thus, we obtain the velocity profile in the overlap layer above rough surfaces as

$$
\tilde{u}=u_{\tau}\left(\frac{1}{\mathcal{K}_{1}} \log \left(y^{+}\right)+B-\Delta U^{+}\left(k_{s \infty}^{+}\right)\right) .
$$

We now assume that we can apply a slip-velocity boundary condition at a flat, lifted virtual wall at $h_{0}>k(x, z)$. It is in this sense that the roughness is considered subgrid. The slip velocity is obtained using (20) evaluated at $y=h_{0}$. Further, to simplify the expression for $\mathcal{K}_{1}$, we recall the assumption that the total shear stress is approximately constant in the overlap region, which allows for modeling the constant value of the total shear stress as the geometric average of its value at the true wall and at the virtual wall, hence, we obtain

$$
\left.\widetilde{u}\right|_{h_{0}}=u_{\tau}\left(\frac{1}{\mathcal{K}_{1}} \log \left(h_{0}^{+}\right)+B-\Delta U^{+}\left(k_{s \infty}^{+}\right)\right) \text {, where } \mathcal{K}_{1}=-\frac{\gamma_{\mathrm{II}} K^{1 / 2}}{2\left(-T_{x y} \mid{\widetilde{l_{s}}}_{s}\right)} .
$$

We can consider $\mathcal{K}_{1}$ as an instantaneous local Kármán constant. Chung and Pullin ${ }^{7}$ estimate the vertical momentum mixing constant as $\gamma_{\text {II }}=0.45$ by matching Townsend's structure parameter-a measure of the relative amount of shear stress to vortex kinetic energy - at the interface of regions (I) and (II). Typically, the height of the virtual wall $h_{0}$ is determined as some fraction of the first grid size, and presently $h_{0}=0.18 \Delta_{y}$ is used.

In summary, the near-wall SGS model, for subgrid roughness, is implemented as follows: for every cell adjacent to the walls, (16) is solved for $u_{\tau}$ and hence the friction velocity is calculated dynamically. Then the log-relation (21) is used to obtain the slip velocity at the lifted virtual wall at $y=h_{0}$. These processes are coupled with the outer LES in two ways: first, some terms in the RHS of the ODE (16) as well as the shear stress $\left.T_{x y}\right|_{\tilde{e}_{s}}$ necessary for evaluating $\mathcal{K}_{1}$ are supplied by the outer LES. The resultant slip velocity at the lifted virtual wall provides the LES with the Dirichlet boundary conditions. The roughness functions are left in implicit form to accept any roughness type and empirical formula appropriate to the specific use of the model. The explicit form of roughness function is discussed subsequently. 


\section{E. Wall-normal velocity boundary condition}

We have thus far considered wall-parallel slip velocity to incorporate surface roughness effects, yet it is also possible to account for wall-normal velocity at the virtual wall with roughness taken into consideration. The filtered continuity equation is given by

$$
\frac{\partial \tilde{u}}{\partial x}+\frac{\partial \tilde{v}}{\partial y}+\frac{\partial \tilde{w}}{\partial z}=0 .
$$

By integrating this continuity equation from the actual wall to the virtual wall height and assuming that the filtered span-wise velocity is zero, we obtain the vertical velocity at the virtual wall as

$$
\left.\widetilde{v}\right|_{h_{0}}=-h_{0} \frac{\partial\langle\tilde{u}\rangle}{\partial x} .
$$

Utilizing an inner-scaling argument, which is similar to how (15) is obtained for the wall-parallel velocity boundary condition, leads to the expression for the wall-normal velocity boundary condition,

$$
\left.\widetilde{v}\right|_{h_{0}}=-h_{0} \frac{\partial u_{\tau}}{\partial x}\left(\frac{\left.\tilde{u}\right|_{h_{0}}}{u_{\tau}}-k_{s \infty}^{+} \frac{\partial \Delta U^{+}}{\partial k_{s \infty}^{+}}\right) .
$$

In the studies of Flores and Jiménez ${ }^{35}$ and Orlandi and Leonardi ${ }^{34}$ where the roughness effects are modeled by finite velocity perturbation at wall boundaries, the wall-normal velocity is observed to have influences on the flow. Here, the wall-normal velocity has been calculated passively in the simulation at each wall point to identify the relative magnitudes of the wall-normal velocity to wall-parallel slip velocity. It has been found that the wall-normal velocity is three to four orders of magnitude smaller than the wall-parallel slip velocity, thus, the vertical velocity at the virtual wall is omitted in our simulations.

\section{F. Roughness function}

The explicit forms of roughness functions have been of interest in theoretical, experimental, and computational studies of wall bounded turbulent flows. They are influenced by both universal roughness effects (as in Townsend ${ }^{22}$ ) and potentially by the individual choice of roughness types according to their geometric profile. A comprehensive summary of roughness functions is presented by Jiménez. ${ }^{18}$ Presently roughness is quantified in terms of geometric roughness $k_{s \infty}$ and the well established Colebrook empirical formula (25) is used for the roughness function (Jiménez ${ }^{18}$ ):

$$
\Delta U^{+}=\kappa^{-1} \log \left(1+0.26 k_{s \infty}^{+}\right) .
$$

The geometric roughness in wall units can be expressed in terms of the Reynolds number $R e_{\tau}$ and relative roughness $\epsilon$,

$$
k_{s \infty}^{+}=\epsilon R e_{\tau}, \quad \epsilon \equiv \frac{k_{s \infty}}{\delta}, \quad R e_{\tau}=\frac{u_{\tau} \delta}{v} .
$$

When the Colebrook formula is incorporated in our wall model, the second term of the denominator in (16) becomes

$$
\begin{aligned}
k_{s \infty}^{+} \frac{\partial \Delta U^{+}}{\partial k_{s \infty}^{+}} & =\frac{1}{\mathcal{K}_{1}} \frac{0.26 k_{s \infty}^{+}}{1+0.26 k_{s \infty}^{+}}, \\
& =\frac{1}{\mathcal{K}_{1}} \frac{0.26 \epsilon \delta u_{\tau} / v}{1+0.26 \epsilon \delta u_{\tau} / v} .
\end{aligned}
$$

Here, the locally and dynamically determined values of the Kármán constant $\mathcal{K}_{1}$ and friction velocity $u_{\tau}$ are obtained from the outer LES and wall model, respectively. We remark that (16) together with (27) shows dependency on $u_{\tau}$ in both the denominator and numerator. Additionally, with the introduction of the Colebrook formula, the log-relation (21) takes the following form:

$$
\left.\widetilde{u}\right|_{h_{0}}=u_{\tau}\left(\frac{1}{\mathcal{K}_{1}} \log \left(h_{0}^{+}\right)+B-\frac{1}{\mathcal{K}_{1}} \log \left(1+0.26 k_{s \infty}^{+}\right)\right) .
$$


We remark that the roughness function is a model input supplied by experiment, theory, low Reynolds number DNS, or some other means. This gives our LES a semi-empirical character. Nonetheless, the use of a roughness function is confined to the wall model only, which presently operates over $0.5 \%-1 \%$ of the half channel height $\delta$, depending on resolution. The choice of roughness function should be determined by specific surface type.

\section{RESULTS}

The incompressible LES-modeled Navier Stokes equations are solved numerically for a roughwall channel of dimensions $L_{x} \times L_{y} \times L_{z}$ (stream-wise, wall-normal, and span-wise directions, respectively). The fully conservative skew-symmetric form of the nonlinear terms is employed. The span-wise direction is treated spectrally, making use of the periodic boundary conditions, which then allow for using a pseudo-spectral method for the nonlinear term in this direction with a $p_{1}$ th-order Fourier exponential filter, which mimics the $2 / 3$ rule, in order to prevent aliasing errors (Gottlieb and $\mathrm{Shu}^{46}$ and Chung and Pullin ${ }^{7}$ ). The other two directions (stream-wise and wall-normal directions) are discretized via fourth-order finite difference on a staggered grid with Dirichlet boundary conditions at the top and bottom walls and periodic boundary conditions in the stream-wise direction in accordance with an infinite channel. All the terms are treated explicitly except for the wall-normal viscous diffusion terms. The temporal discretization is achieved via a low-storage third-order semiimplicit Runge-Kutta method (Spalart et al. ${ }^{47}$ ) with a fractional step method at each stage obtained through approximate LU decomposition (Perot ${ }^{48}$ and Inoue and Pullin ${ }^{8}$ ). The incompressibility is enforced by the pressure-Poisson equation, which reduces to a series of two-dimensional Helmholtz equations and ultimately a series of linear equations. Such reduction of the Helmholtz equation is achieved through Fourier expansions in the span-wise direction and cosine transforms in the other directions (Inoue and Pullin ${ }^{8}$ ). The boundary treatment, in part, follows Morinishi et al. ${ }^{49}$ where a ghost-point scheme extends the grid points beyond the computational domain so that a consistent stencil can be employed throughout. The ghost-point scheme is designed to globally conserve mass and momentum and is effectively equivalent to a one-sided finite-difference scheme at the walls. ${ }^{8}$

Prior to discussing the present high Reynolds number LES, we make contact with the DNS results of Hoyas and Jiménez ${ }^{50}$ for smooth-wall channel flow at $R e_{\tau} \sim 2000$ and Flores and Jiménez, ${ }^{35}$ who performed channel-flow DNS at $R e_{\tau} \sim 630$ where the non-slip and impermeability velocity boundary conditions were perturbed by zero-mean fluctuations in order to model the effect of roughness. The mean velocity profiles and stream-wise turbulent intensities are shown in Fig. 1. For the rough-wall cases, the DNS profile at $R e_{\tau}=632$ and $k_{s \infty}^{+}=129$ was obtained by perturbing both the stream-wise and wall-normal velocities. Also shown is our LES profile at $k_{s \infty}^{+}=100$. Only a few LES data points overlap the DNS. This is because our LES has the requirement that the roughness be subgrid. Since $k_{s \infty}^{+}=\epsilon R e_{\tau}$, then $k_{s \infty}^{+}=100, R e_{\tau}=632$ would give $\epsilon \approx 0.16$ which violates this condition with the present uniform grid. Our LES uses $R e_{\tau}=2 \times 10^{4}$ and $\epsilon=5 \times 10^{-3}$. Also included on the plot is the Colebrook-corrected log law. The DNS result at $k_{s \infty}^{+}=129$ lies on the LES results and the associated Colebrook corrected log law at $k_{s \infty}^{+}=100$. This discrepancy in the equivalent sand roughness is because Flores and Jiménez obtained the mean velocity shift by fitting their mean velocity profile to a roughness modified $\log$ law, which was then used to solve for the equivalent sand roughness. Additionally, they reported that the value of the mean velocity shift varied by $\sim 15 \%$ depending on the value of the Kármán constant, leading to varying values of equivalent sand roughness. Note that while they used $\kappa=0.41$ for their analysis, our LES suggests $\kappa=0.37$. The comparison of the stream-wise intensity (Fig. 1(b)) features only the rough-wall cases and shows reasonable agreement between the DNS result of Flores and Jiménez and our LES results. A relatively large discrepancy is observed closer to the wall and is attributed to the present wall-modeled LES, which does not resolve the near-wall region.

Large eddy simulations are implemented with two different resolutions over a range of the relative roughness $\epsilon=k_{s \infty} / \delta$ as well as the Reynolds number $\operatorname{Re}_{\tau}: 650,2 \times 10^{3}, 4 \times 10^{3}, 2 \times$ $10^{4}, 2 \times 10^{5}, 2 \times 10^{6}, 1 \times 10^{7}, 2 \times 10^{7}$, and $2 \times 10^{8}$, which are summarized in Table I. The upper limit of the roughness is imposed by the assumption that the roughness of interest is subgrid, and hence it should not exceed the height of the lifted virtual wall, $k_{s \infty}<h_{0}$. This implies that the 

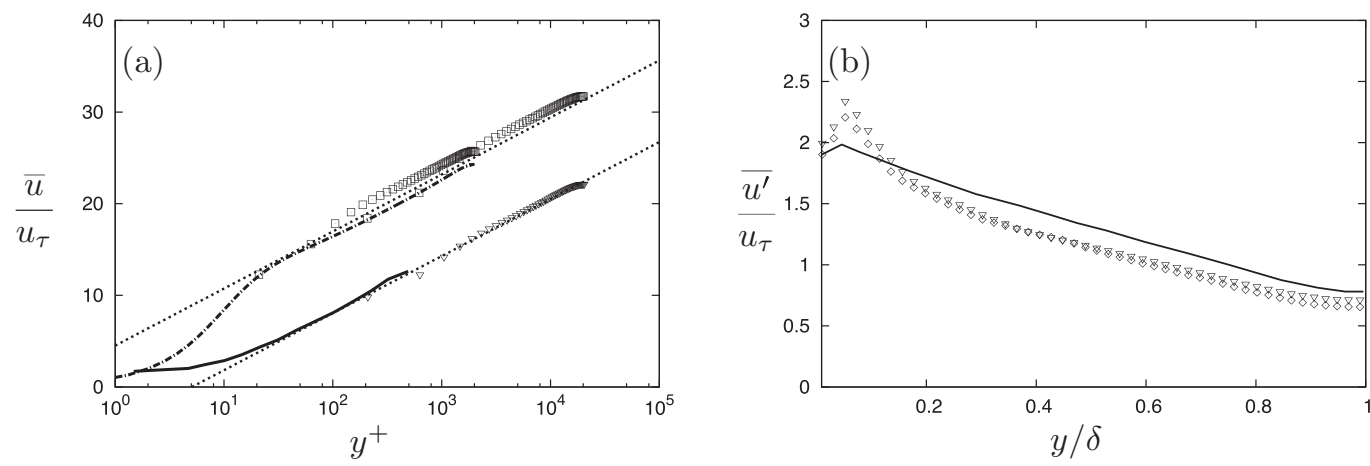

FIG. 1. Comparison between LES and DNS (Refs. 35 and 50): (a) mean velocity profiles and (b) stream-wise turbulence intensity. $\square:$ LES $\epsilon=0$ (smooth), $\nabla:$ LES $k_{s \infty}^{+}=100$, dash-dot line: DNS smooth, solid line: DNS $k_{s \infty}^{+}=129$, dotted line: $\log$ law with the Colebrook formula associated with LES (only in (a)).

upper limit of the relative roughness is resolution dependent. With the current wall model, this upper limit of the relative roughness is estimated as $\epsilon<0.18 \Delta_{z} / \delta$. In this section, five major results are discussed: friction factor from LES, empirical equivalent Moody diagram, mean velocity profile, universal asymptotic velocity defect profile, and turbulent statistics.

\section{A. Friction factor from LES}

Channel flows are driven by the mean pressure gradient. One way of measuring this is to define the friction factor. For both channel and pipe flows, friction factor is defined as the mean pressure gradient scaled by the dynamic pressure. The friction factor is defined by

$$
\begin{aligned}
f & \equiv \frac{-2 \delta d p / d x}{\rho u_{b}^{2}}=\frac{2 \tau_{w}}{\rho u_{b}^{2}}, \\
& =2 \frac{\overline{u_{\tau}^{2}}}{u_{b}^{2}},
\end{aligned}
$$

TABLE I. Summary of LES simulations. The first letter of each case indicates the resolution (high or low). The first and second number of each case indicate different realizations of $\epsilon$ and $R e_{\tau}$, respectively.

\begin{tabular}{lccccccccc}
\hline \hline Case & $R e_{\tau}$ & $L_{x} / \delta$ & $L_{y} / \delta$ & $L_{z} / \delta$ & $N_{x}$ & $N_{y}$ & $N_{z}$ & $\epsilon$ & $\epsilon R e_{\tau}$ \\
\hline L0.1-L0.6 & $650-2 \times 10^{6}$ & 32 & 2 & 8 & 192 & 48 & 48 & 0 & 0 \\
L1.1-L1.6 & $650-2 \times 10^{6}$ & 32 & 2 & 8 & 192 & 48 & 48 & $1 \times 10^{-4}$ & $0.065-200$ \\
L2.1-L2.6 & $650-2 \times 10^{6}$ & 32 & 2 & 8 & 192 & 48 & 48 & $5 \times 10^{-4}$ & $0.325-1000$ \\
L3.1-L3.6 & $650-2 \times 10^{6}$ & 32 & 2 & 8 & 192 & 48 & 48 & $1 \times 10^{-3}$ & $0.65-2000$ \\
L4.1-L4.6 & $650-2 \times 10^{6}$ & 32 & 2 & 8 & 192 & 48 & 48 & $2 \times 10^{-3}$ & $1.3-4000$ \\
L5.1-L5.6 & $650-2 \times 10^{6}$ & 32 & 2 & 8 & 192 & 48 & 48 & $5 \times 10^{-3}$ & $3.25-10000$ \\
H0.1-H0.9 & $650-2 \times 10^{8}$ & 32 & 2 & 8 & 384 & 96 & 96 & 0 & 0 \\
H1.6 & $2 \times 10^{6}$ & 32 & 2 & 8 & 384 & 96 & 96 & $1 \times 10^{-6}$ & 2 \\
H2.6 & $2 \times 10^{6}$ & 32 & 2 & 8 & 384 & 96 & 96 & $5 \times 10^{-6}$ & 10 \\
H3.5-H3.6 & $2 \times 10^{5}-2 \times 10^{6}$ & 32 & 2 & 8 & 384 & 96 & 96 & $1 \times 10^{-5}$ & $2-20$ \\
H4.5 & $2 \times 10^{5}$ & 32 & 2 & 8 & 384 & 96 & 96 & $5 \times 10^{-5}$ & 10 \\
H5.1-H5.9 & $650-2 \times 10^{8}$ & 32 & 2 & 8 & 384 & 96 & 96 & $1 \times 10^{-4}$ & $0.065-200$ \\
H6.1-H6.9 & $650-2 \times 10^{8}$ & 32 & 2 & 8 & 384 & 96 & 96 & $5 \times 10^{-4}$ & $0.325-1000$ \\
H7.1-H7.9 & $650-2 \times 10^{8}$ & 32 & 2 & 8 & 384 & 96 & 96 & $1 \times 10^{-3}$ & $0.65-2000$ \\
H8.1-H8.6 & $650-2 \times 10^{6}$ & 32 & 2 & 8 & 384 & 96 & 96 & $2 \times 10^{-3}$ & $1.3-4000$ \\
H9.1-H9.9 & $650-2 \times 10^{8}$ & 32 & 2 & 8 & 384 & 96 & 96 & $5 \times 10^{-3}$ & $3.25-10000$ \\
\hline \hline
\end{tabular}




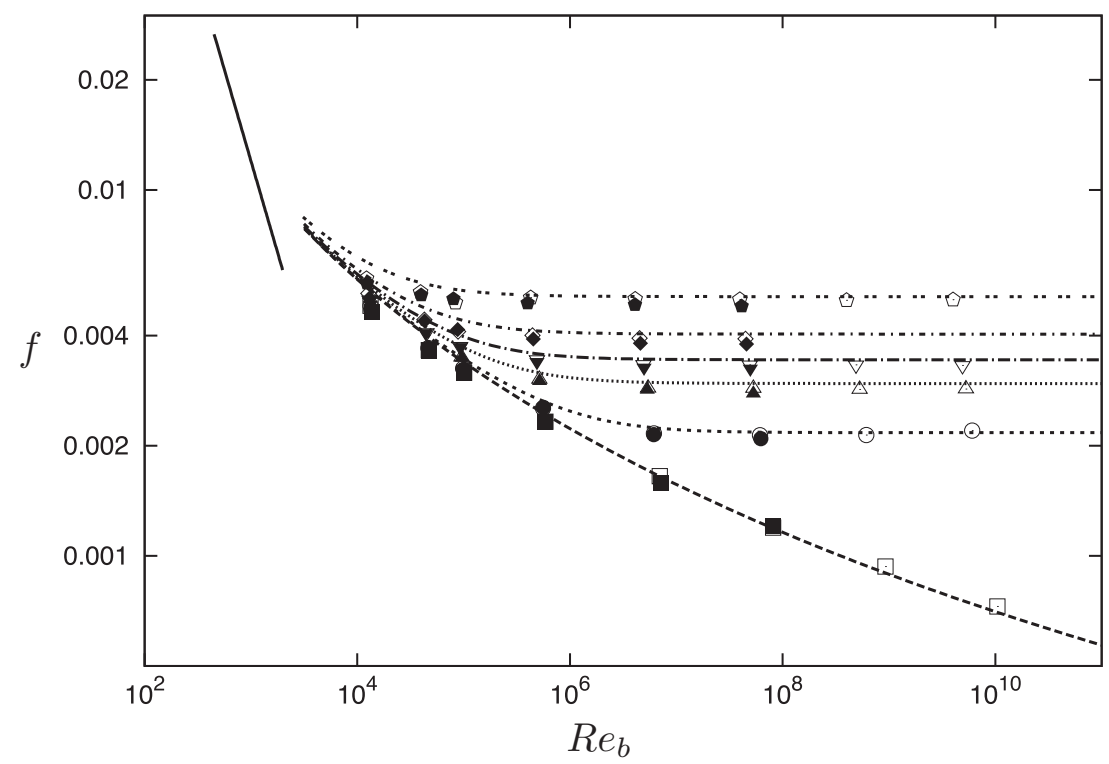

FIG. 2. Friction factor as a function of $R e_{b}$ at various roughness $\epsilon$. Open symbols, high resolution H5.1-H9.6. Solid symbols, low resolution L0.1-L5.6. Symbols indicate $\square: \epsilon=0, \bigcirc: \epsilon=1 \times 10^{-4}, \triangle: \epsilon=5 \times 10^{-4}, \nabla: \epsilon=1 \times 10^{-3}, \diamond: \epsilon=2$ $\times 10^{-3}$, and $\square: \epsilon=5 \times 10^{-3}$. Dashed line, empirical results from Sec. III B. Solid line, theoretical laminar result $f=12 / R e_{b}$.

where $u_{b}$ denotes the channel bulk velocity, and $\tau_{w}$ is the wall-averaged shear stress. We have used $-d p / d x=\tau_{w} / \delta$ for the channel flow and $\tau_{w}=\rho \overline{u_{\tau}^{2}}$, where $\overline{u_{\tau}^{2}}$ is the wall-time-averaged square friction velocity.

From each LES the friction factor $f$ is calculated in accordance with (30) where the time-average of the ratio of wall-averaged $u_{\tau}^{2}$ to bulk velocity $u_{b}^{2}$ at every instance is calculated. Defining the bulk Reynolds number as $R e_{b}=\delta u_{b} / v$ then defines LES-generated points for the function $f=f\left(\epsilon, \operatorname{Re}_{b}\right)$. These are shown in Fig. 2 against $R e_{b}$ for various $\epsilon$. The open and solid symbols distinguish the high resolution cases (H5.1-H9.9) and low resolution cases (L0.1-L5.6), respectively. These results can be viewed as a Moody-like diagram obtained from LES for the given $\Delta U\left(k_{s \infty}^{+}\right)$. Our results cover the fully turbulent regime consisting of smooth, transitionally rough, and fully rough flows. For the smooth-wall case $\epsilon=0$, the friction factor continuously decreases with Reynolds number. In contrast, at fixed $\epsilon>0$, the friction factor decreases with Reynolds number, passing through a Reynolds number dependent phase in the hydrodynamically smooth regime and the transitional regime, and finally approaches a Reynolds number independent phase in the fully rough regime. This asymptotic rough-wall limit is not built into either the wall model or the LES but results from the overall LES-wall-model dynamic calculation.

\section{B. Empirical equivalent Moody diagram}

The original Moody diagram is a representation of friction factor in a pipe as a function of Reynolds number and roughness as introduced by Moody. ${ }^{16}$ It is a graphical representation of an empiricism developed by Colebrook. ${ }^{15}$ Presently we obtain an empirical Moody-diagram for channel flow for comparison with the LES results. We utilize an empirical log law corrected for roughness with the Colebrook formula as

$$
\frac{\tilde{u}(y)}{u_{\tau}}=\frac{1}{\kappa} \log \left(\frac{y u_{\tau}}{v}\right)+B+\frac{\Pi}{\kappa} \mathcal{W}\left(\frac{y}{\delta}\right)-\frac{1}{\kappa} \log \left(1+0.26 \epsilon R e_{\tau}\right),
$$

where here $\kappa$ is the empirical Kármán constant, $B$ is the constant term of the log law, $\Pi$ is the Coles wake factor, $\mathcal{W}(y / \delta)$ is the universal wake function and $u_{\tau} \equiv \sqrt{\overline{\overline{u_{\tau}^{2}}}}$ is here interpreted as an 
average (wall or time-averaged or both) friction velocity. Presently, for simplicity we neglect the wake contribution since it is small for channel flow.

In integrating (31) to obtain $u_{b}$, an approximation to the velocity profile below the log-region is required. Three possible approximations are: (I) The log law is assumed to extend to the wall at $y_{0}^{+}=0$ which is the lower integration limit. (II) The log law is solved for $y_{0}^{+}$assuming $u^{+}\left(y_{0}^{+}\right)=0$ and this result is substituted as the lower integration limit $y_{0}$ such that the inner scaled velocity is zero at the lower integration limit. (III) The piecewise integral of the linear and log-profiles of the velocity with intercept $h_{\nu}^{+}$. For rough walls, this intercept depends on $\epsilon$ and hence it requires solving a relation equating the linear and log-profiles of the velocity, which does not have real solutions for $k_{s \infty}^{+}>14$ approximately. The three methods of computing $u_{b}$ were all implemented and found to give similar results to plotting accuracy. In practice, Method (I) is used owning to its simplicity for which

$$
\begin{aligned}
\frac{u_{b}}{u_{\tau}} & =R e_{\tau}^{-1}\left[\int_{0}^{R e_{\tau}}\left(\kappa^{-1} \log y^{+}+B-\Delta U^{+}\right) d y^{+}\right], \\
& =\frac{1}{\kappa}\left(\log \left(R e_{\tau}\right)-1\right)+B-\Delta U^{+} .
\end{aligned}
$$

Evaluating the integral and substituting it into (30) together with the Colebrook formula $\Delta U^{+}\left(k_{s \infty}^{+}\right)$ then gives an empirical $f=f\left(\epsilon, R e_{\tau}\right)$. Since $R e_{b}=R e_{\tau} u_{b} / u_{\tau}$, Eq. (32) can also be used to evaluate $R e_{b}=R e_{b}\left(\epsilon, R e_{\tau}\right)$ from which curves of $f=f\left(\epsilon, R e_{b}\right)$ can be constructed. These are shown in Fig. 2 in addition to the LES results. The empirical results and LES results are well matched with some difference for low $R e_{b}$. For the highest relative roughness case of $\epsilon=5 \times 10^{-3}$, the equivalent sand roughness height actually exceeds the virtual wall height, thus violating one of the assumptions of subgrid roughness. Nonetheless the friction factors are robustly calculated and match the empirical curve reasonably well. With the lower resolutions, the friction factors at all the Reynolds number and roughness are slightly under-predicted. For this reason, most results in this paper are drawn from the higher resolution cases $\mathrm{H} 0.1-\mathrm{H} 9.9$.

The limiting case of the empirical friction factor in the fully rough asymptotic regime can be obtained by taking the limit of (32) when $R e_{\tau} \rightarrow \infty$ with $\epsilon$ fixed. This gives

$$
\begin{aligned}
\lim _{R e_{\tau} \rightarrow \infty} f & =\lim _{R e_{\tau} \rightarrow \infty} 2\left[\frac{1}{\kappa}\left(\log \left(R e_{\tau}\right)-1\right)+B-\frac{1}{\kappa} \log \left(1+0.26 \epsilon R e_{\tau}\right)\right]^{-2}, \\
& =\left\{\begin{array}{cl}
\frac{2}{\left(B-\kappa^{-1}(\log (0.26 \epsilon)+1)\right)^{2}} & \text { for } \epsilon \neq 0 \\
0 & \text { for } \quad \epsilon=0 .
\end{array}\right.
\end{aligned}
$$

For $\epsilon>0$, this limit is shown in Fig. 3 together with the LES estimate obtained at $R_{b}=2 \times 10^{6}$ with good agreement for our high-resolution LES. At lower resolution, the LES underestimates the limit compared to the higher resolution case.

\section{Mean velocity profiles}

The inner-scaled, mean velocity profiles from the LES results show collapse on constant values of $\epsilon R e_{\tau}$ ranging from 2 to 2000 as illustrated in Fig. 4 for $\left\{\epsilon: 0,1 \times 10^{-6}, 5 \times 10^{-6}, 1 \times 10^{-5}, 5 \times 10^{-5}\right.$, $\left.1 \times 10^{-4}, 1 \times 10^{-3}\right\}$ and $\left\{\operatorname{Re}_{\tau}: 2 \times 10^{4}, 2 \times 10^{5}, 2 \times 10^{6}, 2 \times 10^{7}, 2 \times 10^{8}\right\}$. At each value of Reynolds number, the inner-scaled mean velocity profile is shifted downwards as the relative roughness is increased, and this is expected in rough-wall flows where surface drag is increased by roughness elements. The wall effect is visible, although it is relatively small. The sensitivity of the LES to the height of the virtual wall, i.e., the value of $h_{0} / \Delta_{y}$ has been investigated for $0.09<h_{0} / \Delta_{y}$ $<0.36$. It has been shown that the centerline velocity of each case does not depart from the case with the currently adopted value of 0.18 by more than $5 \%$, being consistent with the observation of Chung and Pullin. ${ }^{7}$ 


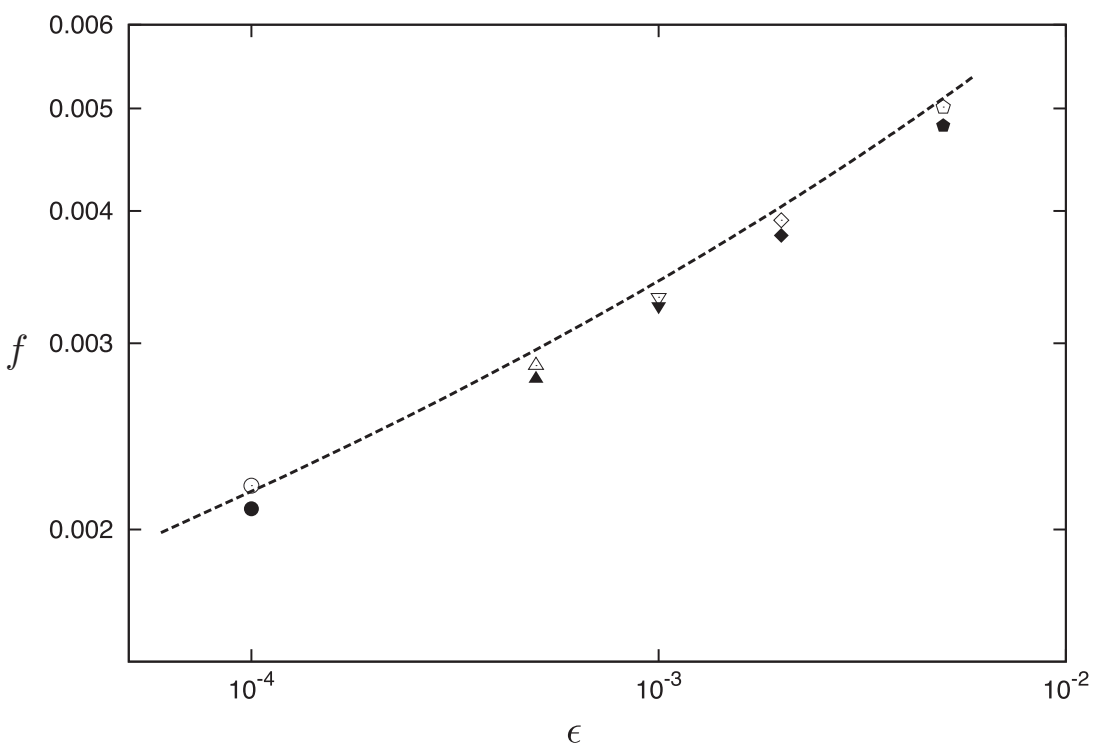

FIG. 3. High Reynolds number limit of friction factor for rough walls. Open symbols, fully rough regime (H5.6, H6.6, H7.6, H8.6, H9.6). Solid symbols, fully rough regime (L1.6, L2.6, L3.6, L4.6, L5.6). Solid line, mathematical limit of empirical result.

Also plotted in Fig. 4 are empirical velocity profiles, where the empirical Kármán constant is taken as $\kappa=0.37$. We recall from (31) that with a roughness correction, the log law intercepts are shifted downwards, and that the amount of the shift solely depends on the value of $k_{s \infty}^{+}=\epsilon R e_{\tau}$. This is consistent with the LES results in Fig. 4. For the smooth-wall cases $(\epsilon=0)$, the LES fits well with the empirical results with its intercept $B=11-\log (11) / 0.37=4.5$. This value of the intercept is in agreement with our wall model definition in (18) and, due to our higher Reynolds number regime, is consequently lower than the classic value of $\approx 5$ (as in the numerical study at $R e_{\tau}=620$ conducted by Moin and $\mathrm{Kim}^{51}$ ). For rough walls, the LES results also closely align themselves with the empirical profiles having intercept $11-\log (11) / 0.37-\log \left(1+0.26 \epsilon R e_{\tau}\right) / 0.37$ for each value

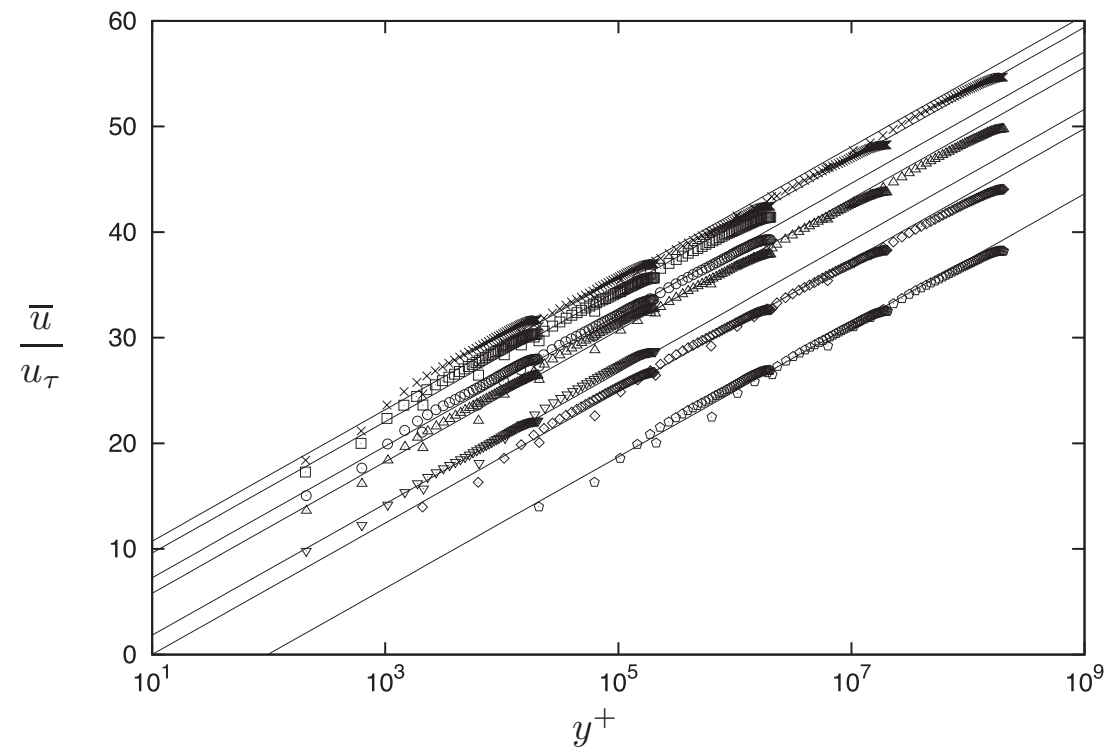

FIG. 4. Mean velocity profiles. Open symbols, LES results $\times: \epsilon \operatorname{Re}_{\tau}=0, \square: \epsilon \operatorname{Re}_{\tau}=2, \bigcirc: \epsilon \operatorname{Re}_{\tau}=10, \triangle: \epsilon \operatorname{Re}_{\tau}=20, \nabla: \epsilon \operatorname{Re}_{\tau}$ $=100, \diamond: \epsilon e_{\tau}=200$, and $\square: \epsilon e_{\tau}=2000$. Dashed lines, empirical $\log$ law with Colebrook's formula. 
of $\epsilon R e_{\tau}$, thus providing a quantification of the momentum loss due to roughness from the perspective of an inner-scaled mean velocity profile. We note that the LES profiles span the transitionally rough regime.

\section{Universal asymptotic velocity deficit profile}

When wall surfaces are rough there exists a universal asymptotic velocity profile in the limit of $R e_{\tau} \rightarrow \infty$. To demonstrate this we take the limit of the empirical log law with Colebrook's roughness correction by allowing $R e_{\tau} \rightarrow \infty$ and we arrive at a universal, asymptotic velocity-deficit profile for rough walls. Our analysis starts by considering the ratio of the mean velocity to the centerline velocity, $\widetilde{u}(y) / u_{c}$, where $u_{c}$ is the channel centerline velocity. We write this as (34) and consider both ratios on the RHS separately:

$$
\frac{\tilde{u}(y)}{u_{c}}=\frac{\tilde{u}(y)}{u_{\tau}} \frac{u_{\tau}}{u_{c}}
$$

The first ratio $\tilde{u}(y) / u_{\tau}$ is given by the empirical log law with Colebrook's roughness correction (31) and its limit is expressed in terms of the wall-normal distance and relative roughness $\epsilon$ in the limit of large Reynolds number as

$$
\begin{aligned}
\lim _{R e_{\tau} \rightarrow \infty} \frac{\tilde{u}(y)}{u_{\tau}} & =\lim _{R e_{\tau} \rightarrow \infty} \frac{1}{\kappa} \log \left(y^{+}\right)+B-\frac{1}{\kappa} \log \left(1+0.26 \epsilon R e_{\tau}\right), \\
& =\frac{1}{\kappa} \log \left(\frac{y}{\delta}\right)+B-\frac{1}{\kappa} \log (0.26 \epsilon) .
\end{aligned}
$$

The second ratio $u_{\tau} / u_{c}$ is given by the log law with Colebrook formula evaluated at the centerline $y$ $=\delta$, whose limit of high Reynolds number is obtained as

$$
\begin{aligned}
\lim _{R e_{\tau} \rightarrow \infty} \frac{\tilde{u}(\delta)}{u_{\tau}}=\lim _{R e_{\tau} \rightarrow \infty} \frac{u_{c}}{u_{\tau}} & =\lim _{R e_{\tau} \rightarrow \infty} \frac{1}{\kappa} \log \left(\frac{\delta u_{\tau}}{v}\right)+B-\frac{1}{\kappa} \log \left(1+0.26 \epsilon R e_{\tau}\right), \\
& =B-\frac{1}{\kappa} \log (0.26 \epsilon) .
\end{aligned}
$$

Using these two ratios (35) and (36) in (34) then gives the ratio of $\tilde{u}(y) / u_{c}$ in the high Reynolds number limit as

$$
\frac{\tilde{u}(y)}{u_{c}}=\frac{\kappa^{-1} \log (y / \delta)+B-\kappa^{-1} \log (0.26 \epsilon)}{B-\kappa^{-1} \log (0.26 \epsilon)},
$$

which can then be rearranged to obtain the deficit form

$$
K\left(1-\frac{\tilde{u}(y)}{u_{c}}\right)=-\frac{1}{\kappa} \log \left(\frac{y}{\delta}\right), \text { where } K=B-\kappa^{-1} \log (0.26 \epsilon) .
$$

Equation (38) is a universal roughness-modified velocity deficit scaled in outer variables $u_{c}$ and $\delta$. We remark that, since when $R e_{\tau} \rightarrow \infty$, we expect $u_{\tau} \sim u_{c} / K$, then this is consistent with Townsend's hypothesis. ${ }^{22}$ Figure 5 shows the LES results plotted in the form of the LHS of (38) that are to be compared with the RHS of (38) using $\kappa=0.37$. The left figure (Fig. 5(a)) includes all LES data regardless of the Reynolds number or roughness level (H5.1-H9.6). These cases include profiles for the transitionally rough regime up to the fully rough regime, and they are observed to deviate from the log-profile suggested by (38). In Fig. 5(b), however, the profiles are limited to those that show collapse on the RHS of (38), including the most rough case of $\epsilon=5 \times 10^{-3}$ at $R e_{\tau}=2000$ as well as the least rough cases of $\epsilon=1 \times 10^{-4}$ at $R e_{\tau}=2 \times 10^{6}$. Physically, the collapse can be explained by Townsend's hypothesis, noting that the LHS of (38) recovers the standard defect form in the limit of high Reynolds number. Commonly true for the collapsed profiles is that the values of $k_{s \infty}^{+}$are above the order of unity, which approximately corresponds to the fully rough regime in our friction factor plot (Fig. 2). This supports that given a sufficiently large value of $k_{s \infty}^{+}$, the high-Reynolds-number- 

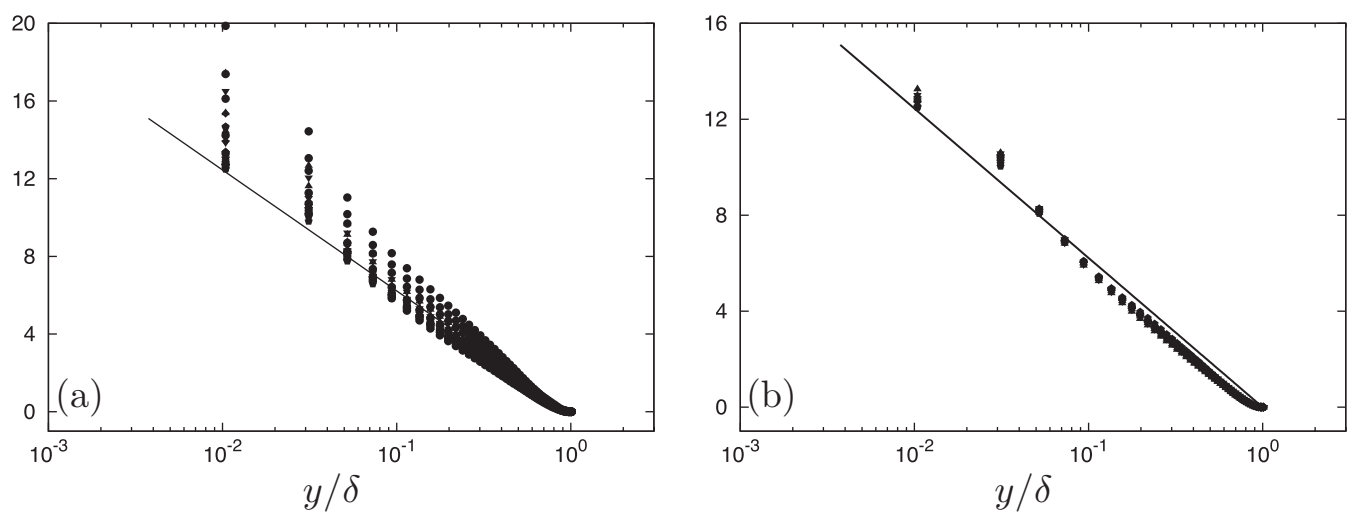

FIG. 5. Universal asymptotic velocity profiles. (a) $K\left(1-\widetilde{u(y)} / u_{c}\right)$ for $\mathrm{H} 5.1-\mathrm{H} 9.6$ and (b) $K\left(1-\widetilde{u(y)} / u_{c}\right.$ ) for $\mathrm{H} 5.5-\mathrm{H} 5.6$, H6.4-H6.6, H7.4-H7.6, H8.3-H8.6, and H9.2-H9.6. Solid line, $\kappa^{-1} \log (y / \delta)$. Symbols, LES results.

asymptotic-limit is in fact universal with a Colebrook-type roughness characterization. Presently we have used an empirical roughness function with $\Delta U^{+} \sim \kappa^{-1} \log \left(\epsilon R e_{\tau}\right)$ with $\epsilon=k_{s \infty} / \delta$. Equation (38) suggests an empirical method for determining an equivalent geometric roughness for a given surface defined as $k_{s \infty}$, obtained by determining the value of $K$ that gives the best fit for the experimental velocity profile. Values for $B$ and $\kappa$ would be required.

\section{E. Turbulent statistics}

We now examine turbulent statistics to explore roughness and Reynolds number effects on statistical quantities scaled on the outer length scale $\delta$ and friction velocity $u_{\tau}$. We consider single point statistics above the virtual wall including fluctuations of velocity and Reynolds shear stress while distinguishing between the subgrid and total components of these quantities. First, we examine a set of data where the entire range of $R e_{\tau}$ and $\epsilon$ is present, i.e. smooth, transitionally rough, and fully rough regimes, shown in Fig. 6 . A common observation in all these statistical quantities is their uniform collapse upon scaling with $u_{\tau}$, although a weak dependence on $R e_{\tau}$ and $\epsilon$ over the range of $R e_{\tau}$ from $2 \times 10^{3}$ to $2 \times 10^{8}$ is noted except at $R e_{\tau}=650$, since the wall model is designed for higher Reynolds number. Also notable in Fig. 6 is that the subgrid components of the fluctuations in the span-wise and wall-normal direction as well as in the Reynolds shear stress illustrate an inherent feature of the stretched vortex SGS model, being that the subgrid components are dominant near the wall while their effect is felt to a lesser extent as we approach the centerline. Statistics for smooth-wall cases are now examined below in Sec. III E 1 followed by the rough-wall cases in Sec. III E 2.

\section{Smooth-wall cases: $\epsilon=0$}

A subset of data in Fig. 6 corresponding to the statistics of smooth-wall cases is discussed. Over the full range of Reynolds number $\left(650 \leq R e_{\tau} \leq 2 \times 10^{8}\right)$ in these cases, we observe at least some degree of Reynolds number dependence, yet when only the higher Reynolds number cases are considered, as in Fig. 7, a remarkable improvement in collapse is observed. In addition to their collapse, Reynolds stresses show linear profiles ranging from zero at the channel centerline to unity at the wall.

Recent studies of smooth-wall flows (e.g., Marusic and Kunkel ${ }^{52}$ ) suggest that the stream-wise intensities show a log-profile for the outer part of smooth flat-plate boundary layer flow. The streamwise intensities from our smooth-wall LES are plotted on semi-log axes in Fig. 8. While the profiles show variations for small Reynolds numbers $\left(\operatorname{Re}_{\tau} \leq 2 \times 10^{5}\right)$ as we observe in Fig. 8(a), those at high Reynolds numbers $\left(\operatorname{Re}_{\tau} \geq 2 \times 10^{6}\right)$ collapse well as $\log (y / \delta)$ in the outer flow in Fig. 8(b). The LES cannot probe the near-wall region and so we expect our results to be accurate only for $y / \delta$ 

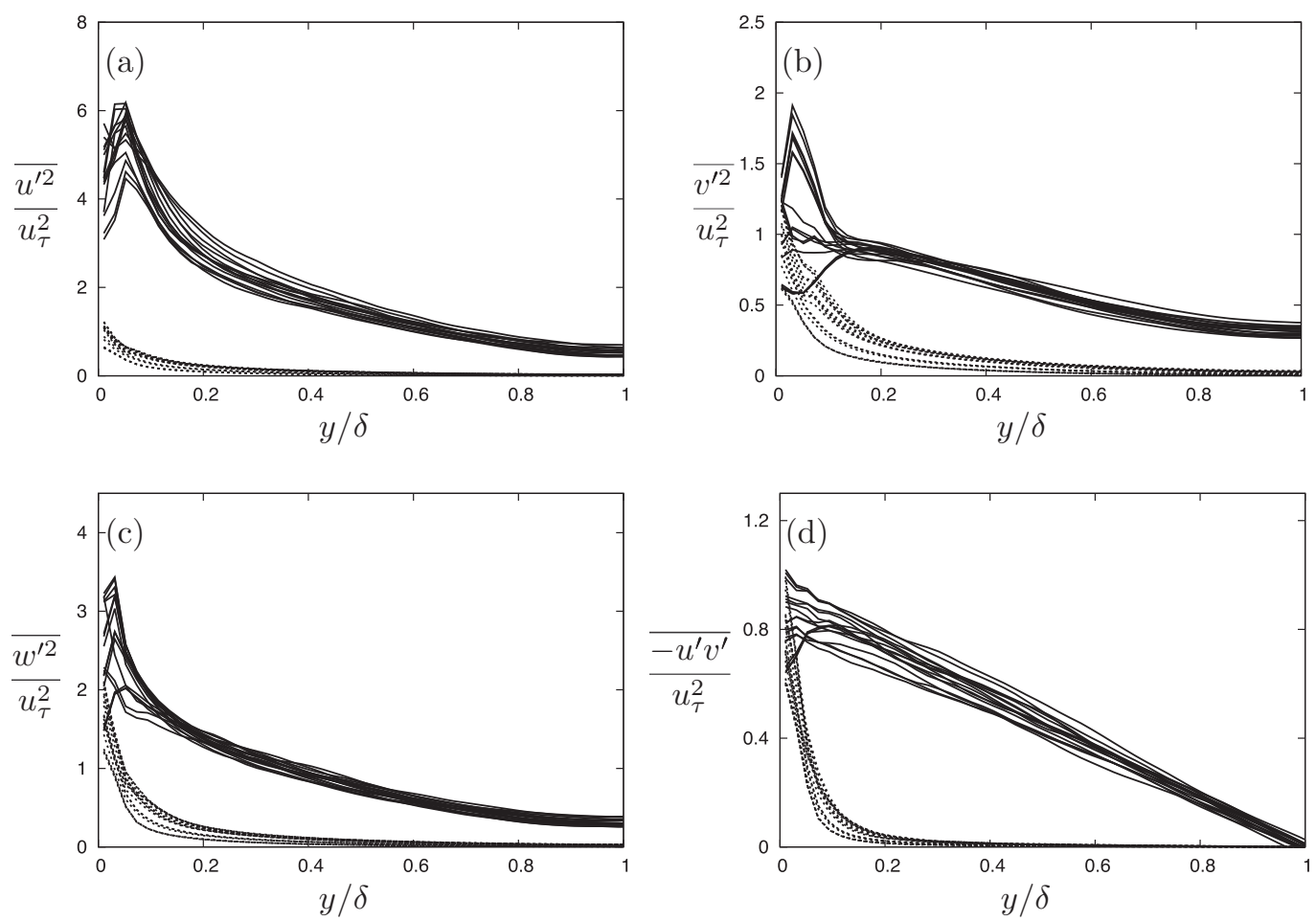

FIG. 6. Turbulent statistics for both smooth and rough walls over full range of Reynolds number. Solid lines, total components. Dashed lines, subgrid components. Reynolds number and relative roughness ranges are $650 \leq R e_{\tau} \leq 2 \times 10^{8}$ and $0 \leq \epsilon \leq 5$ $\times 10^{-3}$.
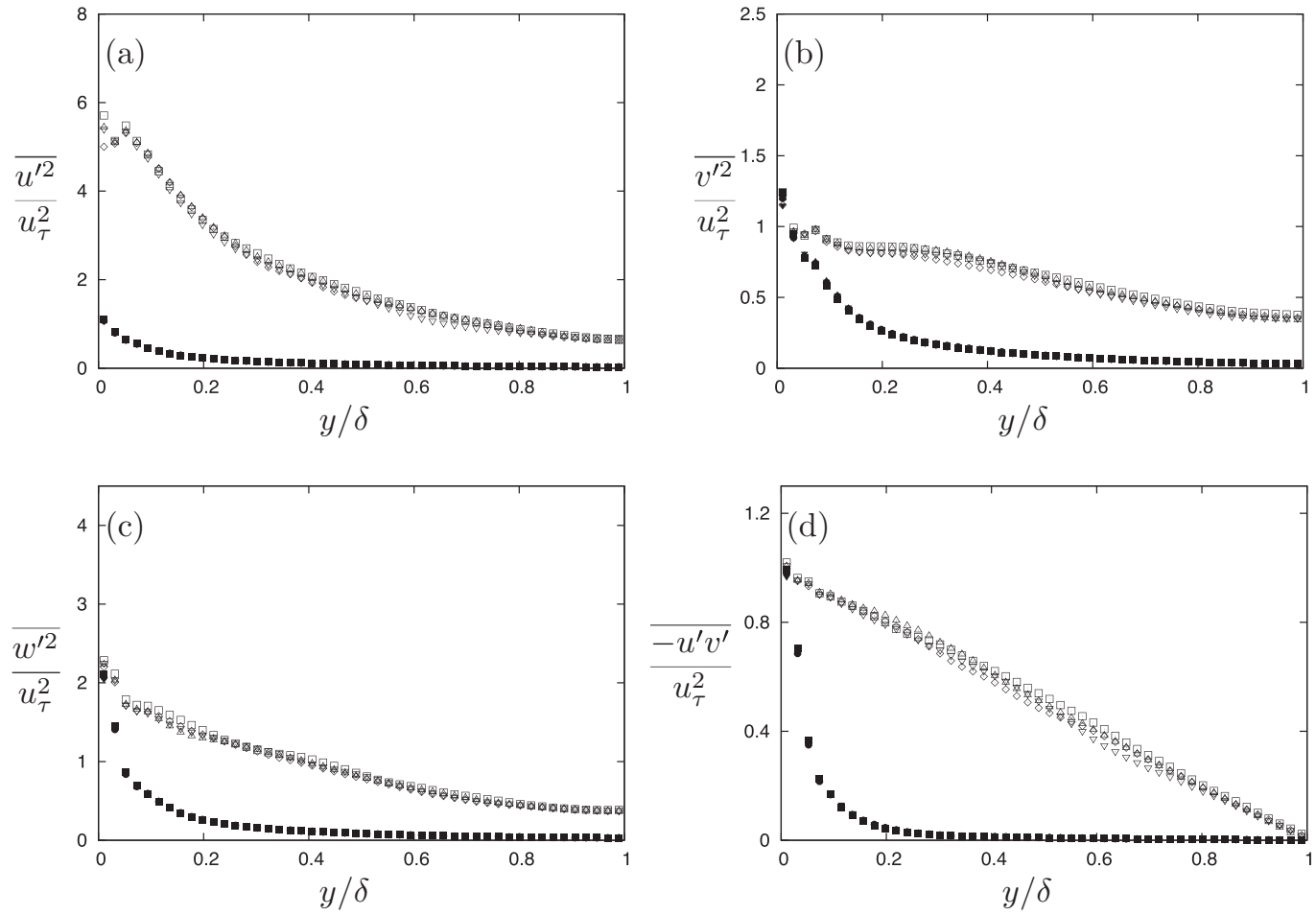

FIG. 7. Turbulent statistics for smooth-wall at high Reynolds number. Open symbols, total components. Solid symbols, subgrid components. $\square: R e_{\tau}=2 \times 10^{6}, \bigcirc: R e_{\tau}=1 \times 10^{7}, \triangle: R e_{\tau}=2 \times 10^{7}$, and $\diamond: R e_{\tau}=2 \times 10^{8}$. 

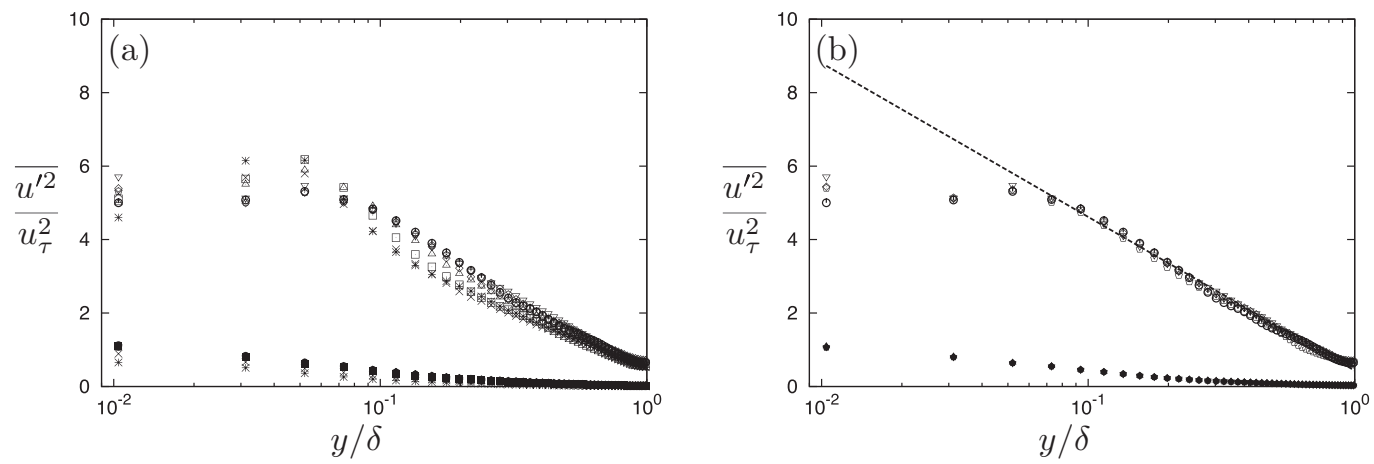

FIG. 8. Stream-wise velocity fluctuations on log-scaled wall-normal distance for smooth-wall cases. (a) Over full range of Reynolds numbers (H0.1-H0.9) and (b) at high Reynolds number. Open symbols, total components. Solid symbols, subgrid components. *: $R e_{\tau}=650, \times: R e_{\tau}=2 \times 10^{3}, \square: R e_{\tau}=2 \times 10^{4}, \bigcirc: R e_{\tau}=2 \times 10^{5}, \triangle: R e_{\tau}=2 \times 10^{6}, \nabla: \operatorname{Re}_{\tau}=1$ $\times 10^{7}, \diamond: R e_{\tau}=2 \times 10^{7}$, and $\checkmark: R e_{\tau}=2 \times 10^{8}$. Dashed line indicates the fitted line according to (39).

$\geq 0.05$. Here the stream-wise fluctuations are bounded above by the sum of a log-profile plus an additional term $T(y / \delta)$ introduced to represent the departure of the profile from the logarithmic line near the centerline

$$
\overline{\frac{u^{\prime 2}}{u_{\tau}^{2}}}=\alpha \log \left(\frac{y}{\delta}\right)+\beta+T(y / \delta) .
$$

Clearly $T(y / \delta) \rightarrow 0$, as $y / \delta \rightarrow 0$. In (39), $\overline{(~)}$ denotes the temporal-planar average, and ( $)^{\prime}$ denotes the fluctuating quantities. Allowing an adequate distance from the wall and centerline, where $T(y / \delta)$ is very small, the coefficients in each case are determined by a least-squares regression, which are then averaged across the range of both $\epsilon$ and $R e_{\tau}$ to obtain $(\alpha, \beta)=(-1.82,0.433)$. The implications of this result are explored in Sec. VI.

\section{Rough-wall cases: $\epsilon \geq 0$}

Smooth-wall data makes up one end of the $\epsilon R e_{\tau}$ range, while at the other end is the rough-wall data, shown in the fully rough regime in Fig. 9. In comparison to the all-encompassing plot of Fig. 6 where smooth, transitionally rough, and fully rough cases are included, the collapse of exclusively fully rough cases improves, with persisting weak dependencies on Reynolds number and roughness. Note that, for the total components of Reynolds stress in the fully rough regime, the plots of higher values of $\epsilon$ tends to lie nearer the origin.

We seek further insight into the logarithmic nature of stream-wise fluctuations by fitting roughwall cases with the functional form in (39), shown in Fig. 10. The left pane (Fig. 10(a)) shows cases including smooth, transitional, and fully rough cases, and the right pane (Fig. 10(b)) emphasizes the asymptotic regime by retaining only fully rough data. The collapse is observed to be improved for fully rough cases. When including all the cases, the coefficients of (39) are calculated to be $(\alpha, \beta)$ $=(-1.63,0.315)$, and $(\alpha, \beta)=(-1.65,0.322)$ when only fully rough cases are included.

\section{DISSIPATION}

To discuss the dissipation $\varepsilon$ we use $\langle Q\rangle, \bar{Q}$, and $\widehat{Q}$ to denote a volume-average over the flow domain, a plane-average over an $(x-z)$ plane and a time-average, respectively. We consider the full Navier-Stokes equations for rough-wall channel flow. It is assumed that the bottom wall shape is $y$ $=k_{l}(x, z)$ and the top wall is $y=2 \delta+k_{u}(x, z)$, where both $k_{l}(x, z)$ and $k_{u}(x, z)$ are periodic in $x$ and $z$ with $\overline{k_{l}(x, z)}=\overline{k_{u}(x, z)}=0$. It is further assumed that any cross section of the channel normal to the $x$-axis has area $A_{x}=L_{x} L_{z}$ that is independent of $x$ so that the channel volume is $V=2 \delta L_{x} L_{z}$. 

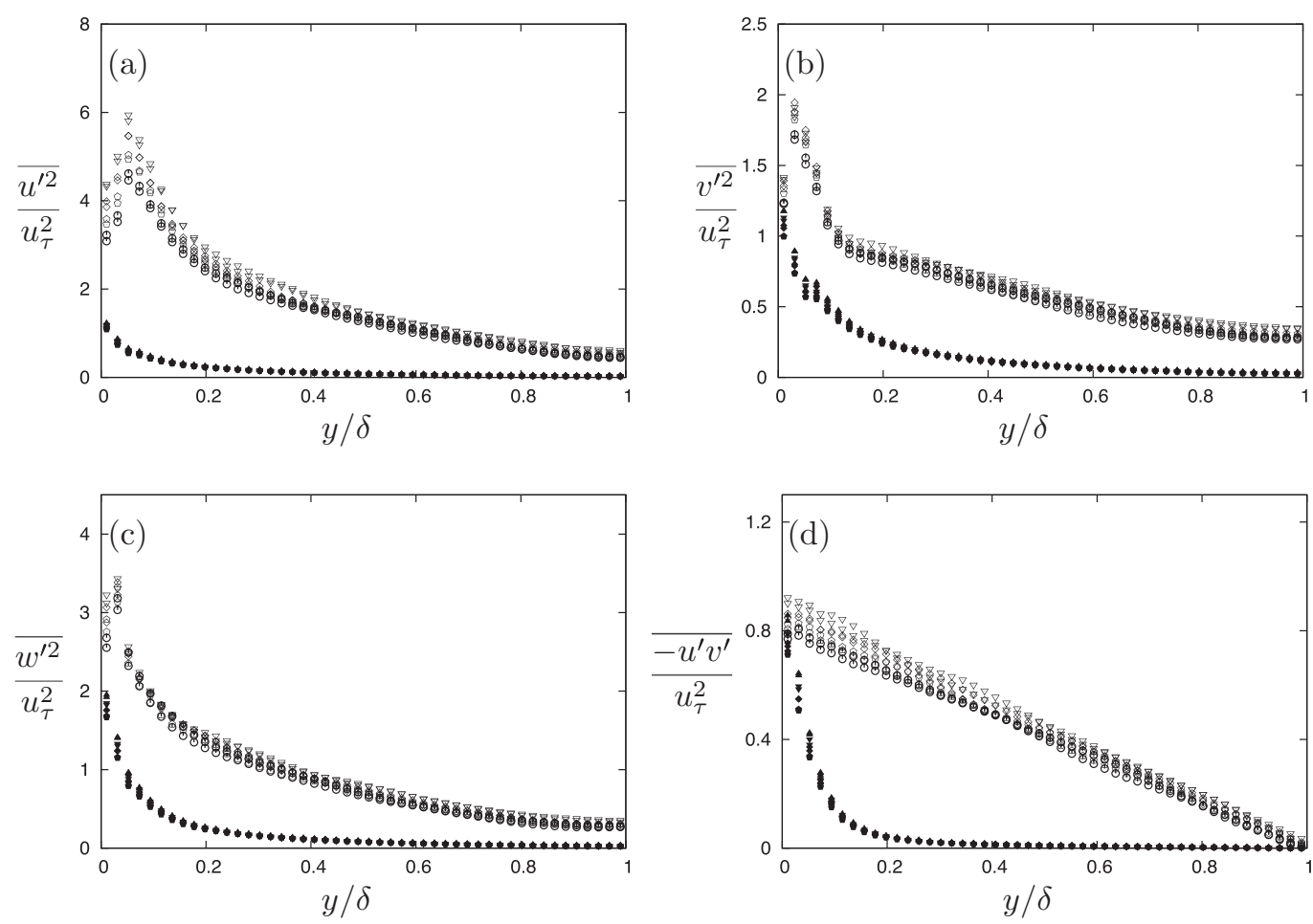

FIG. 9. Turbulence statistics in fully rough regime. Open symbols, total components. Solid symbols, subgrid components. $\triangle: \epsilon=1 \times 10^{-4}, \nabla: \epsilon=5 \times 10^{-4}, \diamond: \epsilon=1 \times 10^{-3}, \diamond: \epsilon=2 \times 10^{-3}$, and $\bigcirc: \epsilon=5 \times 10^{-3}$.

The pressure is expressed as

$$
\begin{aligned}
P & =\mathcal{G}_{j}(t) x_{j}+P^{\prime}\left(x_{1}, x_{2}, x_{3}, t\right), \\
\frac{\partial P}{\partial x_{i}} & =\mathcal{G}_{j}(t) \delta_{i j}+\frac{\partial P^{\prime}}{\partial x_{i}}
\end{aligned}
$$

where $x_{1} \equiv x, x_{2} \equiv y, x_{3} \equiv z, \mathcal{G}_{i}(t)$ is the time-fluctuating mean pressure gradient and $P^{\prime}\left(x_{1}, x_{2}, x_{3}\right.$, $t$ ) is periodic in $x_{1}$ and $x_{3}$. It is further assumed that there is no span-wise mean pressure gradient $\mathcal{G}_{3}(t)=0$, which was enforced in the present code.
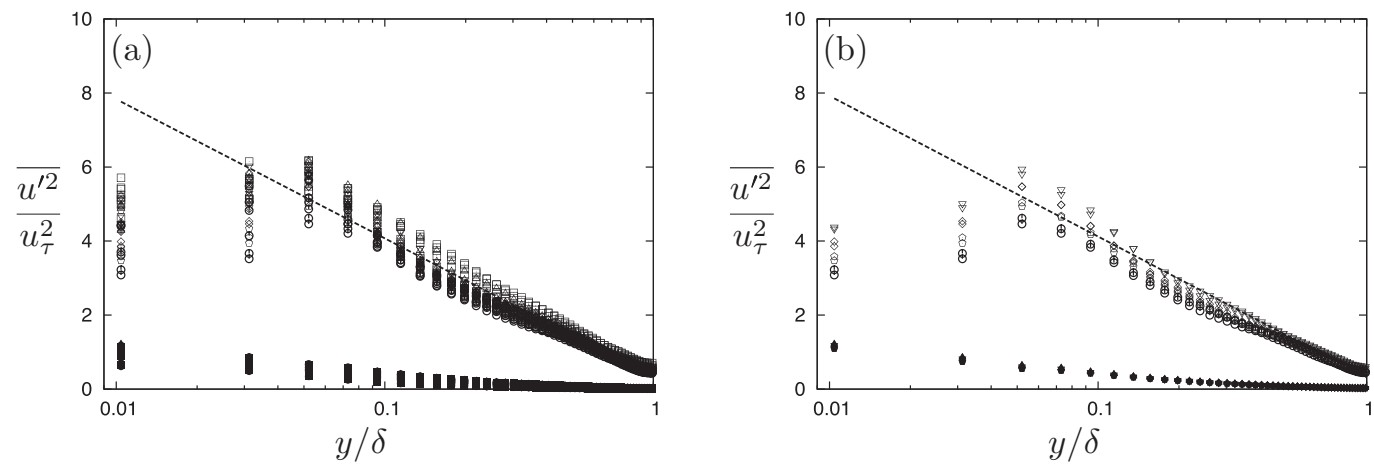

FIG. 10. Stream-wise velocity fluctuation on log-scaled wall-normal distance. (a) Smooth, transitionally rough, and fully rough regime over full range of Reynolds number. (b) Fully rough regime. Open symbols, total components. Solid symbols, subgrid components. $\triangle: \epsilon=5 \times 10^{-4}, \nabla: \epsilon=1 \times 10^{-3}, \diamond: \epsilon=2 \times 10^{-3}$, and $\square: \epsilon=5 \times 10^{-3}$. Dashed lines indicate the fitted line according to (39). 
Volume-averaging the Navier-Stokes momentum equations at fixed time $t$, converting volume integrals to surface integrals (except for the unsteady term) and using the periodic and no-slip boundary conditions gives, for the stream-wise momentum equation

$$
\frac{\partial\left\langle u_{1}\right\rangle}{\partial t}=-\mathcal{G}_{1}(t)-\frac{\overline{u_{\tau}^{2}}}{\delta},
$$

where $\overline{u_{\tau}^{2}}=\overline{\tau_{w} / \rho}$ and $\tau_{w}$ is the drag force per unit area averaged over both walls in the sense of (11) but here extended to a plane-average over both complete channel walls.

The dissipation can be obtained by multiplying the Navier-Stokes equations by $u_{i}$, taking a volume integral and again converting volume to surface integrals. Applying boundary conditions and stream-wise periodicity then leads to

$$
\frac{\partial\left\langle\frac{1}{2} \mathbf{u}_{\mathbf{i}}{ }^{2}\right\rangle}{\partial t}=-\mathcal{G}_{1}(t) u_{1}^{(i n)}-\varepsilon(t),
$$

where $u_{1}^{(i n)}$ is the mean inlet or outlet stream-wise velocity (equal owing to periodicity), and where $\varepsilon(t)=2 \nu\langle\mathbf{S} \cdot \mathbf{S}\rangle$ is the volume-averaged dissipation. The first term on the right-hand side is the pressure work and we note that there is no contribution to this from the generally non-zero but fluctuating mean span-wise velocity at the channel side planes since $\mathcal{G}_{3}=0$, and no contribution from the wall-normal motions owing to the wall boundary conditions and the co-ordinate-normal side planes.

Since $A_{\mathrm{x}}(x)$ is constant then from continuity and periodicity of $u_{3},\left\langle u_{1}\right\rangle \equiv u_{b}(t)=u_{1}^{(i n)}=u_{1}^{(o u t)}$. Eliminating $\mathcal{G}_{1}(t)$ from (42) and (43) gives

$$
\frac{\partial}{\partial t}\left(\left\langle\frac{1}{2} \mathbf{u}^{2}\right\rangle-\frac{1}{2}\left\langle u_{1}\right\rangle^{2}\right)=u_{b}(t) \frac{\overline{u_{\tau}^{2}(t)}}{\delta}-\varepsilon(t) .
$$

We now assume that the channel flow is statistically stationary. The present LES is performed using constant mass flow in which case $u_{b}$ is independent of time but $\mathcal{G}_{1}$ is time-varying and fluctuates about a non-zero mean that, from (42) balances the time-averaged wall drag. On taking a long time average of (44) we obtain

$$
\frac{\delta \widehat{\varepsilon(t)}}{u_{b} \widehat{\widehat{u_{\tau}^{2}}}}=1
$$

or alternatively

$$
\frac{\delta \widehat{\varepsilon(t)}}{u_{b}^{3}}=\frac{f}{2}
$$

Equation (45) also applies to the LES equations if $\varepsilon(t)$ is replaced by

$$
\varepsilon(t)=\left\langle-T_{i j} \widetilde{S_{i j}}+2 v \widetilde{S_{i j}} \widetilde{S_{i j}}\right\rangle,
$$

where the first and second terms are the subgrid and resolved terms, respectively. Figure 11 shows the LHS of (45) plotted against $R e_{b}$, which has been computed from the LES results for various values of Reynolds number and relative roughness. Included are curves based on the total averaged dissipation and also those based only on the viscous contribution.

The total averaged dissipation term in (47) accounts for contributions from regions (I-II), between the actual no-slip walls and the virtual walls, and region (III), the interior LES: see Sec. II D. In region (III), Eq. (47) is used pointwise and integrated over the channel volume. In regions (I-II) below the virtual wall, $\epsilon$ is estimated by approximating the strain-rate tensor $\widetilde{S_{i j}}$ and SGS stress $T_{i j}$ as $d \widetilde{u} / d y$ and $-u_{\tau}^{2}$, respectively, leading to the total dissipation contribution from this region, for one wall

$$
\int_{0}^{h_{0}}\left(-T_{i j} \widetilde{S_{i j}}+2 v \widetilde{S_{i j}} \widetilde{S_{i j}}\right) d y=\int_{0}^{h_{0}}\left(u_{\tau}^{2} \frac{d \tilde{u}}{d y}+\frac{v}{2}\left(\frac{d \tilde{u}}{d y}\right)^{2}\right) d y .
$$




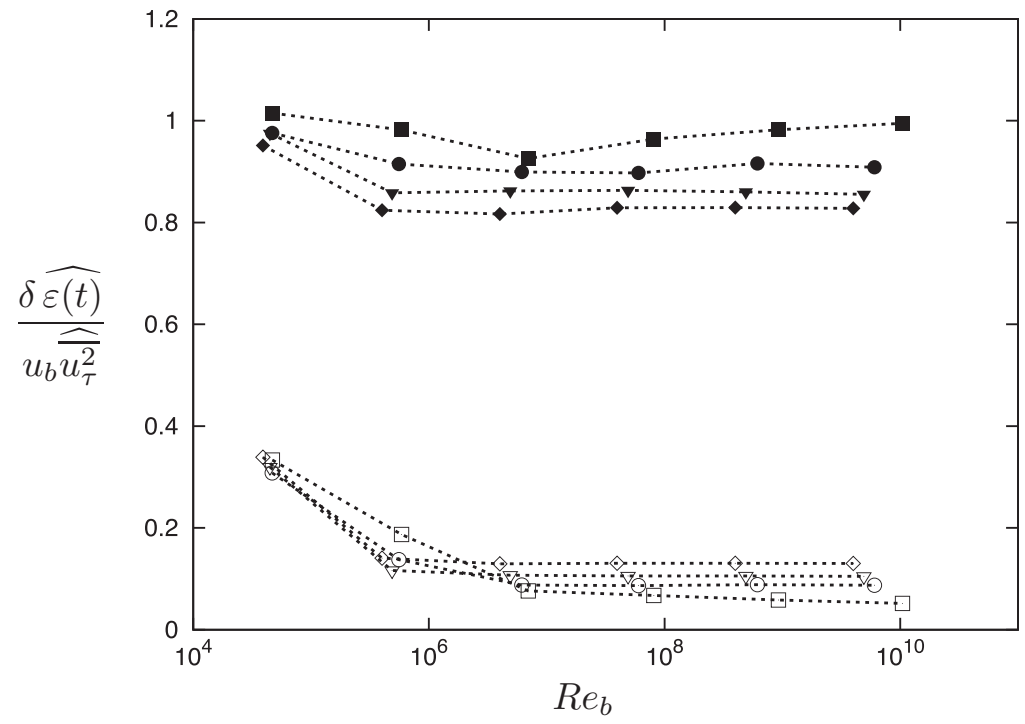

FIG. 11. Left-hand side of (45) at various values of roughness $\epsilon$ and Reynolds number $R e_{\tau}$. LES results are for $\{\epsilon: 0,1 \times$ $\left.10^{-4}, 1 \times 10^{-3}, 5 \times 10^{-3}\right\}$ and $\left\{\operatorname{Re}_{\tau}: 2 \times 10^{3}, 2 \times 10^{4}, 2 \times 10^{5}, 2 \times 10^{6}, 2 \times 10^{7}, 2 \times 10^{8}\right\}$. Open symbols denote resolved contributions. Closed symbols denote both subgrid and resolved contributions. Symbol legend: $\square: \epsilon=0, \bigcirc: \epsilon$ $=1 \times 10^{-4}, \nabla: \epsilon=1 \times 10^{-3}$, and $\diamond: \epsilon=5 \times 10^{-3}$.

While the first term of the integral in the RHS of (48) has the closed form $\left.u_{\tau}^{2} u\right|_{h_{0}}$, the second term is calculated by performing piece-wise integrals over the extent of the linear and log-profiles of mean velocity. The integrands are calculated from the LES using dynamically calculated values of the slip velocity, friction velocity, and local Kármán constant at each wall point. The integrals share a common integration bound, which is calculated as the intercept of the two profiles. Since the log-profile is based on the log law with the Colebrook formula, the intercept varies with $\epsilon$ and $R e_{\tau}$, and thus it is computed through a process of equating the linear relation and the log-relation for each combination of $\epsilon$ and $R e_{\tau}$. In cases where the intercept does not have real solutions $\left(k^{+}>\right.$ 14 approximately), the location where the linear and log-profiles are tangent to each other is used as the common integration bound value.

While the region under the virtual wall occupies only $0.5 \%$ of $\delta$, it in fact accounts for some $60 \%-70 \%$ of the total dissipation. In Fig. 11, the total calculated dissipation scaled on $u_{b}\left\langle\widehat{\overline{u_{\tau}^{2}}}\right\rangle$ is approximately independent of $R e_{b}$ for all smooth and rough-wall cases. When scaled on $u_{b}^{3}$, agreement with (46) is similar to that displayed in Fig. 11. If the limit $R e_{\tau} \rightarrow \infty$ is taken holding $u_{b}$ fixed with $v \rightarrow 0$ then, since the LES and the empirical Moody diagram both support $f \rightarrow 0$ for $\epsilon=0$, it follows that $\widehat{\varepsilon(t)} \rightarrow 0$ in this limit.

\section{FLOW VISUALIZATIONS}

Figure 12 illustrates a color visualization of the local normalized velocity $\widetilde{u(x, y)} / U_{c}$ on a wall-normal $x-y$ plane at different roughness heights and at $R e_{\tau}=2 \times 10^{6}$. Similarly, Fig. 13 shows the modified velocity deficit $K\left(1-\widetilde{u(x, y)} / u_{c}\right)$. Notable in Fig. 12 is that, while the normalized velocities appear similar near the centerline for all the roughness levels, they are distinctly lower towards the wall when larger roughness is present. This is indicative of increased wall shear stress due to wall roughness. In contrast, this reduction is absent from Fig. 13 in which the roughnesscorrected velocity deficit appears largely independent of $\epsilon$. Recall from Sec. III D that the velocity deficit profiles collapse in the high Reynolds number asymptotic limit.

Again at $R e_{\tau}=2 \times 10^{6}$, Fig. 14 shows the instantaneous and locally determined square of the friction velocity $u_{\tau}^{2} / \overline{u_{\tau}^{2}}$ plotted on the bottom physical wall. Note that the color-bar legends are 
(a)

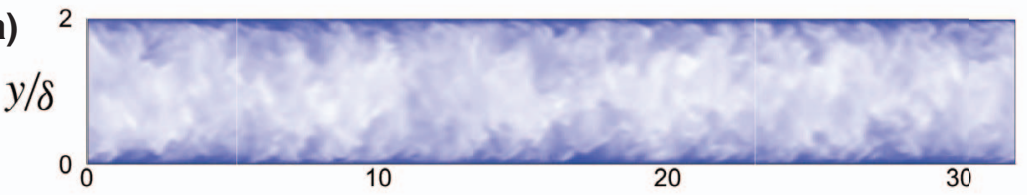

(b)

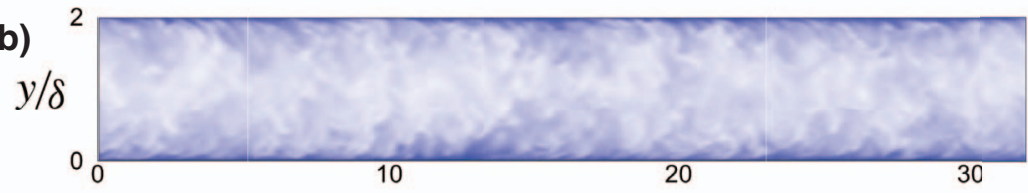

(c)

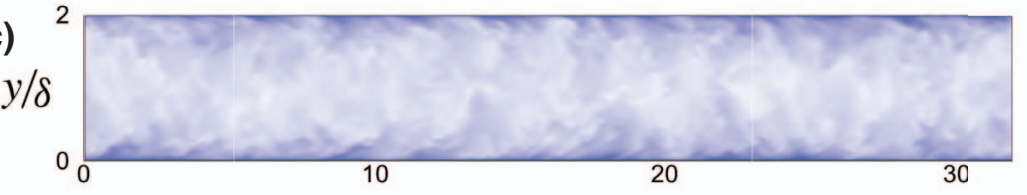

(d)

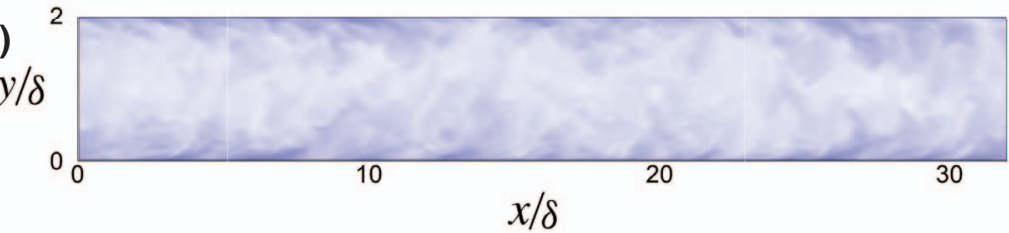

FIG. 12. Local velocity normalized by centerline velocity $\widetilde{u(x, y)} / U_{c}, R e_{\tau}=2 \times 10^{6}$. Roughness heights from the lower to upper panes are, respectively, $\epsilon / \delta=0,1 \times 10^{-4}, 1 \times 10^{-3}, 5 \times 10^{-3}$.

distinct for each plot. By choosing the color-bars to be centered at the mean of each sub-plot data set, the upper and lower bounds can be selected to represent two standard deviations on either side of the mean to highlight the spread of the data. The PDF of $u_{\tau}^{2}(x, y) / \overline{u_{\tau}^{2}}$ is shown in Fig. 15(a), where we observe that the spread of the data broadens with increasing roughness. In addition, the long stream-wise oriented streak-like structures in the smooth-wall case (lowest pane) are shortened with increasing roughness. The DNS results of Orlandi and Leonardi ${ }^{34}$ showed a shortening of near-wall $\left(y^{+} \approx 12\right)$ vorticity streaks for several wall roughness types of a $2 \mathrm{D}$ and $3 \mathrm{D}$ nature. They attribute this effect to the increased intensity of turbulent disturbances produced by the roughness compared to a smooth wall. Their roughness height was $20 \%$ of the half channel height, with $k^{+} \approx 10$, making
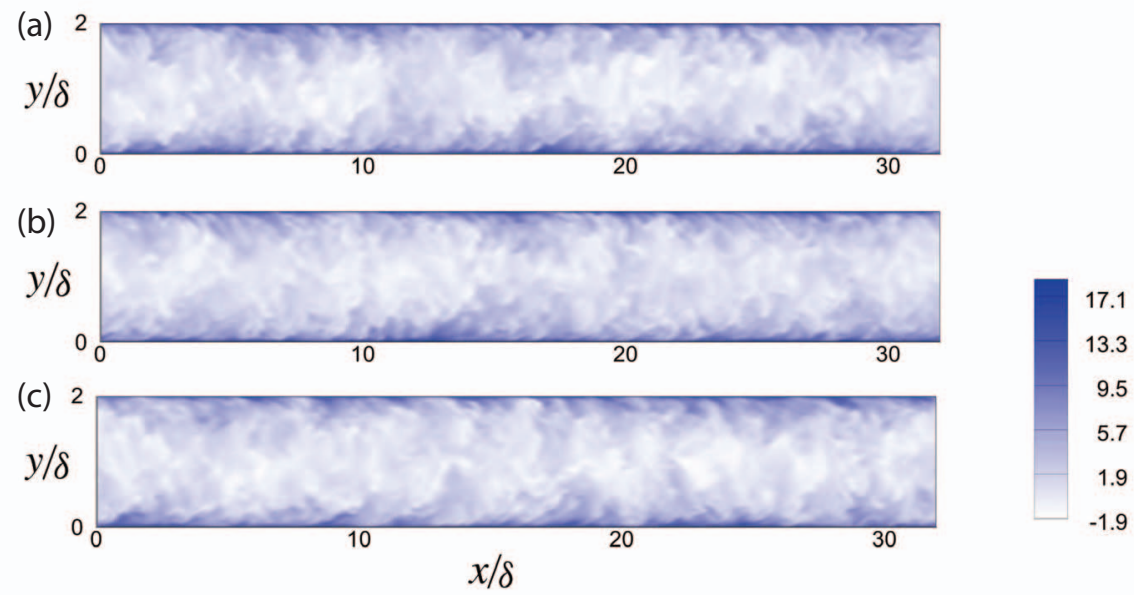

FIG. 13. Local velocity deficit $K\left(1-\widetilde{u(x, y)} / u_{c}\right), R e_{\tau}=2 \times 10^{6}$. Roughness heights from the lower to upper panes are, respectively, $k / \delta=1 \times 10^{-4}, 1 \times 10^{-3}$, and $5 \times 10^{-3}$. 
(a)
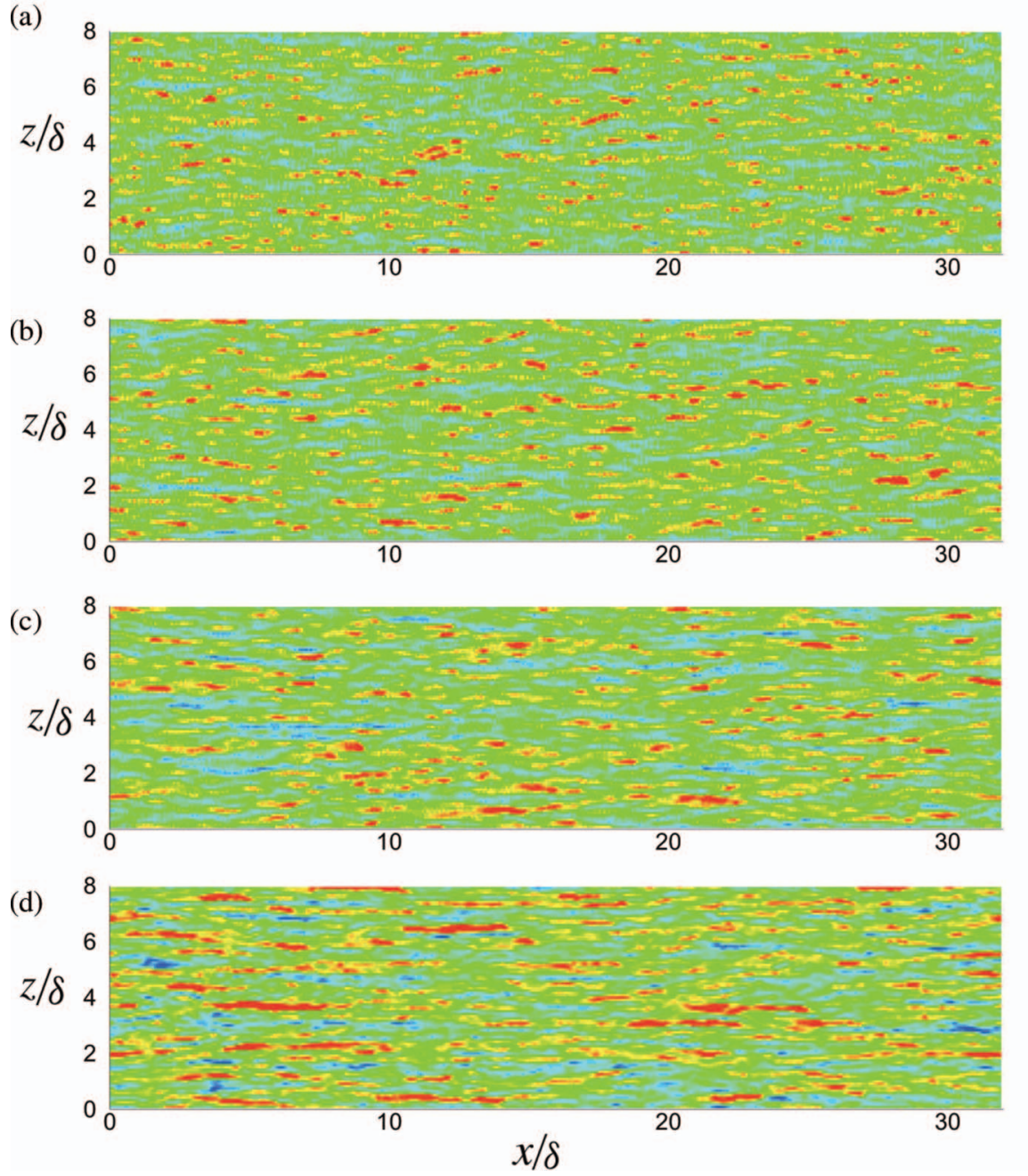

FIG. 14. Distribution of $u_{\tau}^{2} / \overline{u_{\tau}^{2}}$ on the bottom wall. (a) $\epsilon=5 \times 10^{-3}$, (b) $\epsilon=1 \times 10^{-3}$, (c) $\epsilon=1 \times 10^{-4}$, and (d) smooth $(\epsilon=0)$.

it difficult to make any direct comparisons with the present results. In a separate DNS study, Flores and Jiméne $z^{35}$ observed length-reduction of near-wall streaks and explained the phenomenon as a result of the disturbed near-wall cycle in the buffer-layer caused by the dynamics of the roughness sublayer, where the layer typically extends to 2-5 times the roughness height. However, our virtual wall locates above the buffer layer and the relation of the observed streak-like structures to the near-wall cycle is unclear. Finally, the instantaneous friction factor fields, $f(x, y)=2 u_{\tau}^{2}(x, y) / u_{b}^{2}$ on the bottom wall are discussed. Owing to our simulation conditions wherein the mass flow is kept constant, the instantaneous contours of friction factor (not shown) and $u_{\tau}^{2} / \overline{u_{\tau}^{2}}$ (Fig. 14) show inherent differences in the contour levels but otherwise identical profiles at each roughness level. However, it is apparent in the PDF of the friction factor shown in Fig. 15(b) that the mean value of friction factor is not constant and increases with $\epsilon$, which is expected of the rough-wall cases as seen in the friction factor plot (Fig. 2).

\section{DISCUSSION}

The results of the present LES agree well with empirical models of the mean-flow velocity and skin friction at large $R e_{\tau}$ and support a stream-wise turbulence intensity that scales on $u_{\tau}^{2}$ with 

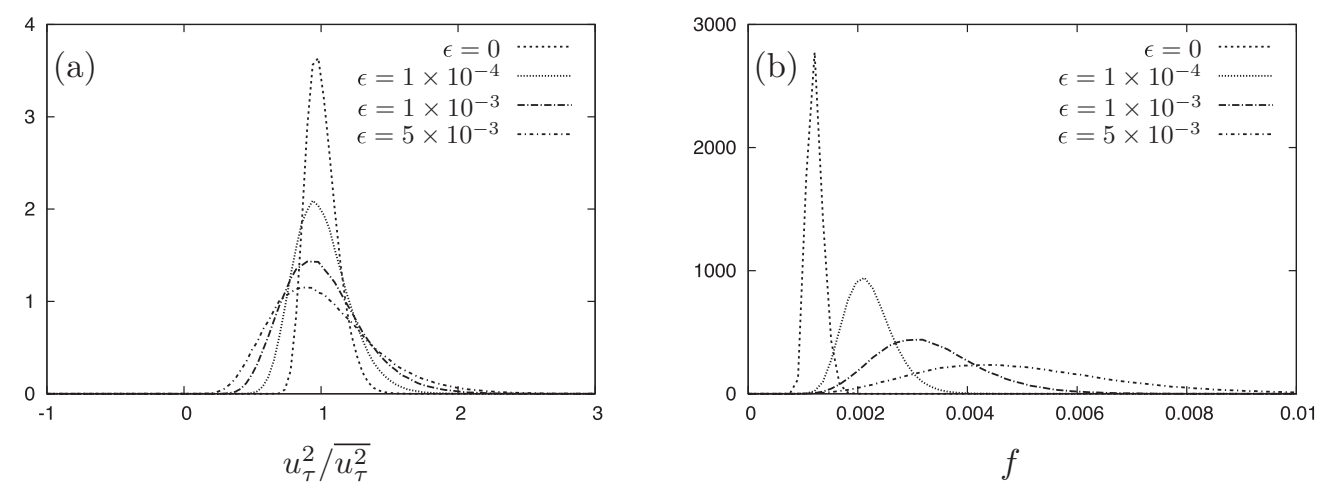

FIG. 15. Probability density function of (a) friction velocity and (b) friction factor for $R e_{\tau}=2 \times 10^{6}$ and $\epsilon=0,1$ $\times 10^{-4}, 1 \times 10^{-3}$, and $5 \times 10^{-3}$.

logarithmic variation in the outer flow $(y / \delta)$ for both the smooth and rough-wall cases, consistent with Townsend's hypothesis. ${ }^{22}$ We therefore use these results to explore the limit $\operatorname{Re}_{\tau} \rightarrow \infty$ for both rough and smooth-wall channel flow. For the mean velocity, using (31)

$$
\begin{aligned}
\left(\frac{u_{b}}{u_{c}}\right) & =\frac{-\log \left(1+\alpha \epsilon R e_{\tau}\right)+\kappa\left(B+K_{1}\right)+\log \left(R e_{\tau}\right)-1}{-\log \left(1+\alpha \epsilon \operatorname{Re}_{\tau}\right)+\kappa\left(B+K_{2}\right)+\log \left(R e_{\tau}\right)}, \\
K_{1} & =\frac{\Pi}{\kappa} \int_{0}^{1} \mathcal{W}(\xi) d \xi, \quad K_{2}=\frac{\Pi}{\kappa} \mathcal{W}(1),
\end{aligned}
$$

where $u_{c}$ is the mean centerline velocity. If $\epsilon$ is fixed and $R e_{\tau} \rightarrow \infty$, this has the limit

$$
\lim _{R e_{\tau} \rightarrow \infty}\left(\frac{u_{b}}{u_{c}}\right)=\left\{\begin{array}{cc}
\frac{\kappa\left(B+K_{1}\right)-1-\log (\alpha \epsilon)}{\kappa\left(B+K_{2}\right)-\log (\alpha \epsilon)} & \text { for } \epsilon \neq 0 \\
1 & \text { for } \epsilon=0,
\end{array}\right.
$$

which suggests limiting plug-flow for the smooth-wall case.

If it is further assumed that, for $0 \leq \epsilon \ll 1$, the planar-time averaged stream-wise turbulence intensity is bounded above by (39) for $0 \leq y / \delta \leq 1$, that is, $\overline{u^{\prime 2}} / u_{\tau}^{2}$ nowhere in the channel exceeds the right-side of (39), then the ratio of the total stream-wise turbulence intensity to the square of the bulk velocity in the channel flow is bounded by

$$
\frac{\left\langle u^{\prime 2}\right\rangle}{u_{b}^{2}}=\frac{\overline{u^{\prime 2}}}{\overline{u_{\tau}^{2}}} \frac{\overline{u_{\tau}^{2}}}{u_{b}^{2}}=\frac{f}{2}\left(\beta+|\alpha|+\int_{0}^{1} T(\eta) d \eta\right) .
$$

Here, we use \langle\rangle to denote the temporal and volumetric average over the flow domain. Since, for $\epsilon=0, f \rightarrow 0$ when $R e_{\tau} \rightarrow \infty$ and the integral is expected to be finite, then this is asymptotically zero for $\epsilon=0$. For any small but finite $\epsilon$ the volume-averaged, stream-wise turbulence intensity is expected to remain finite and to scale on $f$. Substituting (33) into the right-hand side of (52) gives an approximation for the limiting, average stream-wise turbulent intensity directly in terms of $\epsilon$.

We note that the present LES cannot resolve the near wall region. The above discussion does not preclude, that for $\epsilon=0$, there exists an inner layer of small thickness, where, for example, $\overline{u^{\prime 2}} / u_{b}^{2}$ could remain finite. When $\epsilon=0$ in wall-bounded turbulent flow, it is well known that $\overline{u^{\prime 2}} / \overline{u_{\tau}^{2}}$ has a maximum at around $y^{+}=15$. Experimental studies have indicated, at sufficiently large $R e_{\tau}$ in pipe flow, a second maximum for $\overline{u^{\prime 2}} / \overline{u_{\tau}^{2}}$ further from the wall. ${ }^{12,53}$ McKeon and Sharma ${ }^{54}$ use a critical-layer argument to infer $y_{\mathrm{II}}^{+} \sim R e_{\tau}^{2 / 3}$ for pipe flow while Alfredson et al. ${ }^{55}$ suggest $y_{\mathrm{II}}^{+}=R e_{\tau}^{0.56}$. Let us assume a second peak at $y_{\mathrm{II}}^{+}=A_{1} R e_{\tau}^{a}$, where $A_{1}$ is constant and $0 \leq a$ $<1$. It then follows that $y_{\mathrm{II}} / \delta=A_{1} R e_{\tau}^{a-1}$, so that $y_{\mathrm{II}} / \delta \rightarrow 0$, for $\operatorname{Re}_{\tau} \rightarrow \infty$ with $\epsilon=0$. This is not resolved by the present LES. If however, it is assumed that this peak peels off below (39) at $y \sim y_{\mathrm{II}}$, 
then a simple calculation gives the stream-wise turbulent intensity at the second peak as

$$
\frac{\overline{\frac{u_{\mathrm{II}}^{\prime 2}}{\overline{u_{\tau}^{2}}}}}{{ }_{\tau}} A_{2} \log \left(R e_{\tau}\right)+B_{2},
$$

where $A_{2}$ and $B_{2}$ are constants. Using that, for $\epsilon=0, u_{b} / u_{\tau} \sim \kappa^{-1} \log \left(R e_{\tau}\right)+\mathcal{O}(1)$, supported by the present LES in Fig. 2 up to $R e_{b}=\mathcal{O}\left(10^{10}\right)$, then the ratio

$$
\overline{\frac{u_{\mathrm{II}}^{\prime 2}}{u_{b}^{2}}} \sim 1 / \log \left(R e_{\tau}\right),
$$

and so $\rightarrow 0$ when $R e_{\tau} \rightarrow \infty$. This simple analysis suggests that, when $R e_{\tau} \rightarrow \infty$, even though the maximum stream-wise intensity (assuming that this is at $y_{\mathrm{II}}$ ) becomes unbounded relative to $u_{\tau}$, it is asymptotically zero relative to the outer bulk motion.

For rough-wall flow $\epsilon>0$, then when $R e_{\tau} \rightarrow \infty, k_{s \infty}^{+} \rightarrow \infty$ and all quantities approach finite limits that depend on $f$ and are independent of $\operatorname{Re}_{\tau}$. The present LES, however, uses the Hama roughness function within the wall model. We cannot rule out the possibility that, for resolved-scale wall shapes of small slopes for which $\epsilon>0$ and $k_{s}^{+} \rightarrow \infty$ but there is no local separation, then a smooth-wall-like limit at $R e_{\tau} \rightarrow \infty$ may be appropriate.

\section{CONCLUSIONS}

Our approach to simulate rough-wall channel flows in LES, with subgrid roughness, was to implement the stretched vortex SGS model in the outer region and the Chung and Pullin ${ }^{7}$ wall model was extended using the Hama correction in the near-wall region. The outer LES calculation required no direct modifications. The wall model contained two features that were both affected by roughness; first, the stream-wise momentum equation was combined with roughness-corrected inner scaling, leading to an ODE to be solved numerically and dynamically for the local $u_{\tau}$ at each point on the wall. Hence both $u_{\tau}$ and $k_{s \infty}^{+}$are determined dynamically. With $u_{\tau}$ available and assuming that the near-wall region contains attached eddies, ${ }^{45}$ the velocity profile in the near-wall region was obtained as a log-relation for smooth walls, which was then corrected for roughness to finally be evaluated at the flat lifted virtual wall and used as a wall boundary condition for the outer LES. When applied to both the ODE and log-relation, these roughness corrections employed the Colebrook formula, which expresses the downward shift of velocity profiles as a function of geometric roughness using wall units. The explicit form of the roughness function in this simulation is a model input and it is possible to employ any given roughness function of the form $\Delta U^{+}\left(k_{s \infty}^{+}\right)$or that which can take discrete multi-scale roughness as inputs, that is $\Delta U^{+}\left(k_{s 1}^{+}, k_{s 2}^{+}, k_{s 3}^{+}, \ldots\right)$.

Our LES captured fully developed rough-channel flow in both transitionally rough and fully rough regimes, reaching the maximum bulk velocity based Reynolds number $R e_{b}$ of order $10^{10}$. Our LES results produced a Moody-like diagram for fully developed channel flow including transitionally and fully rough regimes, which showed favorable agreement with an empirical formula obtained from the log law also with the Colebrook roughness correction. The inner-scaled mean velocity profiles showed collapse on constant values of $\epsilon R e_{\tau}$ and were shifted downwards compared to the smooth-wall case by an amount depending on $k_{s \infty}^{+}$. The LES and empirical relation (31) were in agreement for each value of $\epsilon \operatorname{Re}_{\tau}$. The mean velocity profile was also plotted in the outer-scaled deficit form. In the fully rough regime, it collapsed well onto the universal velocity deficit profile obtained by taking the high Reynolds number limit of the empirical relation, providing a means of empirically acquiring $k_{s \infty}$ in a given geometry.

The turbulent statistics consisted of the mean fluctuations of each velocity component as well as the Reynolds shear stress. When including both transitionally and fully rough regimes, weak dependencies on Reynolds numbers were observed for all the cases. Within the fully rough regime, however, improvements in collapse were observed for the velocity fluctuations. At very large Reynolds number the stream-wise turbulence intensities scaled well on $u_{\tau}^{2}$ and $\delta$ for both smooth and rough-wall flows with particularly good collapse for the smooth-wall case. This is in broad agreement with Townsend's hypothesis. Further, for both smooth-wall and rough-wall 
flow in the fully rough regime, the stream-wise turbulence intensities are well described by a $\log (y / \delta)$-profile across almost all of the resolved, outer part of the channel flow. An assumption that these intensities are bounded everywhere by the log-profile leads to the conclusion that the average stream-wise turbulence intensity is proportional to the friction factor. When $R e_{b}$ is extremely large this suggests that the total turbulence intensity approaches a finite value, approximated by (33) for finite roughness, and zero for smooth-wall flow. The dissipation is also proportional to the friction factor and, when scaled with outer variables, becomes independent of $R e_{b}$ when $R e_{b}$ is extremely large. The constant of proportionality is asymptotically zero for smooth-wall flow.

The above suggests that, for the smooth-wall case, the infinite $R e_{b}$ limit is inviscid slip flow without turbulence across almost the whole channel. In this scenario, the near-wall motion associated with $u_{\tau}$ and its fluctuations decline relative to the centerline velocity as the Reynolds number increases. The detailed small-scale physics of this attenuation are certainly of interest but are beyond the scope of this work. The indicated infinite Reynolds number limit for rough-wall channel flow shows a universal, roughness corrected mean-velocity deficit profile together with stream-wise turbulence intensities and total energy dissipation that both scale on the finite friction factor.

Although the present method treats only subgrid roughness, extension to combinations of subgrid roughness together with roughness of order the near-wall grid scale are expected to be straightforward through the implementation of a modified wall-normal velocity proportional to the product of the local slip velocity and the local roughness slope. Owing to the locally dynamic method for calculating the wall friction velocity, the present formulation is not restricted to channel or pipe flow and can, without modification be used to extend smooth-wall LES to include subgrid roughness with and without ${ }^{8}$ pressure gradients.

\section{ACKNOWLEDGMENTS}

The authors acknowledge helpful discussions with B. McKeon.

${ }^{1}$ A. Misra and D. I. Pullin, “A vortex-based subgrid stress model for large-eddy simulation,” Phys. Fluids 9, 2443-2454 (1997).

${ }^{2}$ A. Ferrante, G. Matheou, and P. E. Dimotakis, "LES of an inclined sonic jet into a turbulent crossflow at Mach 3.6," J. Turbul. 12, 1-32 (2011).

${ }^{3}$ H. Pitsch, "Large-eddy simulation of turbulent combustion," Ann. Rev. Fluid Mech. 38, 453-482 (2006).

${ }^{4}$ G. Matheou, A. M. Bonanos, C. Pantano, and P. E. Dimotakis, "Large-eddy simulation of mixing in a recirculating shear flow," J. Fluid Mech. 646, 375 (2010).

${ }^{5}$ H. Foysi and S. Sarkar, "The compressible mixing layer: an LES study," Theor. Comput. Fluid Dyn. 24, 565-588 (2009).

${ }^{6}$ U. Piomelli, "Wall-layer models for large-eddy simulation," Prog. Aero. Sci. 44, 437-446 (2008).

${ }^{7}$ D. Chung and D. I. Pullin, "Large-eddy simulation and wall-modeling of turbulent channel flow," J. Fluid Mech. 631, 281-309 (2009).

${ }^{8}$ M. Inoue and D. I. Pullin, "Large-eddy simulation of the zero-pressure-gradient turbulent boundary layer up to $R e_{\theta}$ $=\mathcal{O}\left(10^{12}\right)$," J. Fluid Mech. 686, 1-27 (2011).

${ }^{9}$ U. Piomelli and E. Balaras, "Wall-layer models for large-eddy simulations," Annu. Rev. Fluid Mech. 34, 349-374 (2002).

${ }^{10}$ J. A. Templeton, G. Medic, and G. Kalitzin, "An eddy-viscosity based near-wall treatment for coarse grid large-eddy simulation," Phys. Fluids 17, 105101 (2005).

${ }^{11}$ J. Templeton, M. Wang, and P. Moin, "A predictive wall model for large-eddy simulation based on optimal control techniques," Phys. Fluids 20, 065104 (2008).

${ }^{12}$ I. Marusic, B. J. McKeon, P. A. Monkewitz, H. M. Nagib, A. J. Smits, and K. R. Sreenivasan, "Wall-bounded turbulent flows at high Reynolds numbers: Recent advances and key issues," Phys. Fluids 22, 065103 (2010).

${ }^{13}$ B. J. Mckeon, J. Li, W. Jiang, J. F. Morrison, and A. J. Smits, "Further observations on the mean velocity distribution in fully developed pipe flow," J. Fluid Mech. 501, 135-147 (2004).

${ }^{14}$ J. Nikuradse, "Strömungsgestze in rauhen rohren," VDI-Forschungsh. 4(361), 1-22 (1933).

${ }^{15}$ C. F. Colebrook, "Turbulent flow in pipes, with particular reference to the transition region between the smooth and rough pipe laws," J. Inst. Civil Eng. (London, England) 11, 133-156 (1939).

${ }^{16}$ L. Moody, "Friction factors for pipe flow," Trans. ASME 66, 671-684 (1944).

${ }^{17}$ T. Cebeci and P. Bradshaw, Momentum Transfer in Boundary Layers (Hemisphere Publishing Corporation, 1977).

${ }^{18}$ J. Jiménez, "Turbulent flows over rough walls," Ann. Rev. Fluid Mech. 36, 173-196 (2004).

${ }^{19}$ P. Bradshaw, "A note on 'critical roughness height', and 'transitional roughness'," Phys. Fluids 12, 1611-1614 (2000).

${ }^{20}$ L. Prandtl, "Neuere Ergebnisse der Turbulenzforschung," Z. Ver. Dtsch. Ing. 77, 105-144 (1933).

${ }^{21}$ I. P. Castro, "Rough-wall boundary layers: mean flow universality,” J. Fluid Mech. 585, 469 (2007). 
${ }^{22}$ A. A. Townsend, The Structure of Turbulent Shear Flow, 2nd ed. (Cambridge University Press, 1976).

${ }^{23}$ M. P. Schultz and K. A. Flack, "The rough-wall turbulent boundary layer from the hydraulically smooth to the fully rough regime,"J. Fluid Mech. 580, 381 (2007).

${ }^{24}$ D. W. Bechert, M. Bruse, W. Hage, J. G. T. Van Der Hoeven, and G. Hoppe, "Experiments on drag-reducing surfaces and their optimization with an adjustable geometry," J. Fluid Mech. 338, 59-87 (1997).

${ }^{25}$ F. R. Hama, "Boundary-layer characteristics for smooth and rough surfaces," Trans. Soc. Nav. Arch. Mar. Engrs 62, 333-358 (1954).

${ }^{26}$ M. P. Schultz and K. Flack, “Turbulent boundary layers on a systematically varied rough wall," Phys. Fluids 21, 015104 (2009).

${ }^{27}$ M. A. Shockling, J. J. Allen, and A. J. Smits, "Roughness effects in turbulent pipe flow," J. Fluid Mech. 564, 267-285 (2006).

${ }^{28}$ S. Leonardi, P. Orlandi, and R. A. Antonia, "Properties of d- and k-type roughness in a turbulent channel flow," Phys. Fluids 19, 125101 (2007).

${ }^{29}$ A. Monin, “The atmospheric boundary layer," Ann. Rev. Fluid Mech. 2, 225-250 (1970).

${ }^{30}$ J. Counihan, "Adiabatic atmospheric boundary layers: a review and analysis of data from the period 1880-1972," Atmos. Environ. 9, 871-905 (1975).

${ }^{31}$ M. M. Metzger and J. Klewicki, "A comparative study of near-wall turbulence in high and low Reynolds number boundary layers," Phys. Fluids 13, 692-701 (2001).

${ }^{32}$ P. Orlandi, S. Leonardi, R. Tuzi, and R. A. Antonia, "Direct numerical simulation of turbulent channel flow with wall velocity disturbances," Phys. Fluids 15, 3587 (2003).

${ }^{33}$ S. Leonardi, P. Orlandi, R. Smalley, L. Djenidi, and R. Antonia, "Direct numerical simulations of turbulent channel flow with transverse square bars on one wall,” J. Fluid Mech. 491, 229-238 (2003).

${ }^{34}$ P. Orlandi and S. Leonardi, "DNS of turbulent channel flows with two- and three-dimensional roughness," J. Turbul. 7, N73 (2006).

${ }^{35}$ O. Flores and J. Jiménez, "Effect of wall-boundary disturbances on turbulent channel flows," J. Fluid Mech. 566, 357 (2006).

${ }^{36}$ A. Nakayama, K. Hori, and R. L. Street, "Filtering and LES of flow over irregular rough boundary," in Proceedings of Summer Program 2004 (Center for Turbulence Research, 2004), pp. 145-156.

${ }^{37}$ A. Anderson and C. Meneveau, "Dynamic roughness model for large-eddy simulation of turbulent flow over multiscale, fractal-like rough surfaces," J. Fluid Mech. 679, 288-314 (2011).

${ }^{38}$ D. I. Pullin and P. G. Saffman, "Vortex dynamics in turbulence," Ann. Rev. Fluid Mech. 30, 31-51 (1997).

${ }^{39}$ T. S. Lundgren, "Strained spiral vortex model for turbulent fine structure," Phys. Fluids 25, 2193-2203 (1982).

${ }^{40}$ D. I. Pullin, "A vortex-based model for the subgrid flux of a passive scalar," Phys. Fluids 12, 2311-2319 (2000).

${ }^{41}$ D. I. Pullin and T. S. Lundgren, "Axial motion and scalar transport in stretched spiral vortices," Phys. Fluids 13, 2553-2563 (2001).

${ }^{42}$ P. A. O'Gorman and D. I. Pullin, “The velocity-scalar cross spectrum of stretched spiral vortices,” Phys. Fluids 15, 280-291 (2003).

${ }^{43}$ M. R. Head and P. Bandyopadhyay, "New aspects of turbulent boundary-layer structure," J. Fluid Mech. 107, 297-338 (1981).

${ }^{44}$ S. K. Robinson, "Coherent motions in the turbulent boundary layer," Annu. Rev. Fluid Mech. 23, 601-639 (1991).

${ }^{45}$ T. B. Nickels, I. Marusic, S. Hafez, N. Hutchins, and M. S. Chong, "Some predictions of the attached eddy model for a high Reynolds number boundary layer," Philos. Trans. R. Soc. A 365, 807-822 (2007).

${ }^{46}$ D. Gottlieb and C. Shu, "On the Gibbs phenomenon and its resolution," SIAM Rev. 39, 644-668 (1997).

${ }^{47}$ P. R. Spalart, R. D. Moser, and M. M. Rogers, "Spectral methods for the Navier-Stokes equations with one infinite and two periodic directions," J. Comp. Phys. 96, 297-324 (1991).

${ }^{48}$ J. Perot, “An analysis of the fractional step method,” J. Comput. Phys. 108, 51-58 (1993).

${ }^{49}$ Y. Morinishi, T. Lund, O. Vasilyev, and P. Moin, "Fully conservative higher order finite difference schemes for incompressible flow," J. Comput. Phys. 143, 90-124 (1998).

${ }^{50} \mathrm{~S}$. Hoyas and J. Jiménez, "Scaling of the velocity fluctuations in turbulent channels up to $R e_{\tau}=2003$," Phys. Fluids $\mathbf{1 8}$, 011702 (2006)

${ }^{51}$ P. Moin and J. Kim, "Numerical investigation of turbulent channel flow," J. Fluid Mech. 118, 341-377 (1982).

${ }^{52}$ I. Marusic and G. J. Kunkel, "Streamwise turbulence intensity formulation for flat-plate boundary layers," Phys. Fluids 15, 2461 (2003).

${ }^{53}$ J. F. Morrison, B. McKeon, W. Jiang, and A. Smits, "Scaling of the streamwise velocity component in turbulent pipe flow," J. Fluid Mech. 508, 99-131 (2004).

${ }^{54}$ B. McKeon and A. Sharma, "A critical-layer framework for turbulent pipe flow," J. Fluid Mech. 658, 336-382 (2010).

${ }^{55}$ P. Alfredsson, A. Segalini, and R. Örlü, "A new scaling for the streamwise turbulence intensity in wall-bounded turbulent flows and what it tells us about the outer peak," Phys. Fluids 23, 041702 (2011). 